\title{
INTERFACING A HIRUDO MEDICINALIS RETZIUS CELL WITH INSULATED GATE OF MOSFET
}

\author{
A Thesis \\ presented to \\ the Faculty of California Polytechnic State University, \\ San Luis Obispo
}

\author{
In Partial Fulfillment \\ of the Requirements for the Degree \\ Master of Science in Biomedical Engineering
}

\author{
by
}

Rachel Marie Smith

December 2017 
(C) 2017

Rachel Marie Smith

ALL RIGHTS RESERVED 
COMMITTEE MEMBERSHIP

TITLE:

Interfacing a Hirudo medicinalis Retzius cell with insulated gate of MOSFET

AUTHOR:

Rachel Marie Smith

DATE SUBMITTED:

December 2017

COMMITTEE CHAIR:

Dr. Robert B. Szlavik, Ph.D. Professor of Biomedical Engineering

COMMITTEE MEMBER:

Dr. David S. Clague, Ph.D. Professor of Biomedical Engineering

COMMITTEE MEMBER:

Dr. Lanny Griffin, Ph.D. Professor of

Biomedical Engineering 


\begin{abstract}
Interfacing A Hirudo Medicinalis Retzius Cell With Insulated Gate Of MOSFET Rachel Marie Smith
\end{abstract}

Much work has been done to study the external stimulation of nervous tissue as well as the transmission of neural signals to electronics. Peter Fromherz was one of the pioneers in this area of electrophysiology, with a series of experiments in the 1990s that aimed to characterize and optimize the interface between neural tissue and transistors. In this thesis, Kurt Sjoberg and I interfaced a Retzius cell isolated from a Hirudo medicinalis ganglion with the insulated gate of a MOSFET. The goal was to see change in membrane potential that could be related Fromherz's original 1991 work. Our experimental setup utilized a classic electrophysiology technique, the current clamp. After varying the amplitude of the stimulating current pulses injected via microelectrode and ensuring the tight seal of the neuronal membrane with the insulated transistor gate, we found evidence of transistor voltage change that was temporally consistent with the elicited action potential of the neuron. 


\section{ACKNOWLEDGMENTS}

To Dr. Szlavik: who has been a guiding force in my academic career since Fall 2014

To Drs. Clague and Griffin: who have agreed to review this thesis, the academic culmination of my time at Cal Poly

To Kurt Sjoberg: who has been a joy to work with on this project

To my family and loved ones: who have tolerated my insanity during this phase of my life

To Kaori Funahashi and the Endurance Cubed team: who motivate me to reach my full potential as an age-group triathlete

To the Kaiser Permanente Clinical Engineering team: who give me countless reasons to look forward to my professional career 


\section{TABLE OF CONTENTS}

Page

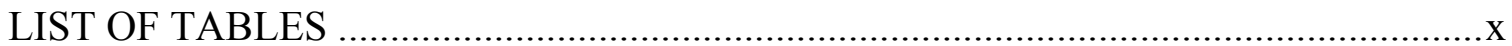

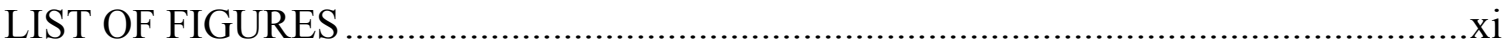

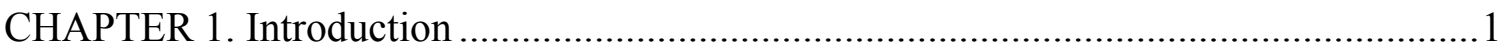

1.1 Motivation........................................................... 1

1.2 Previous Work...........................................................

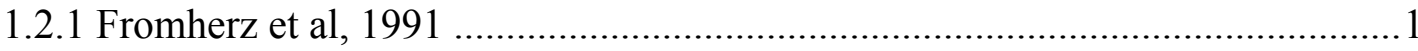

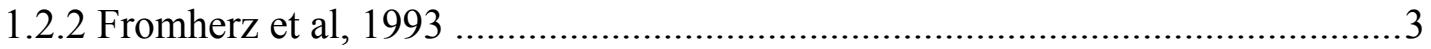

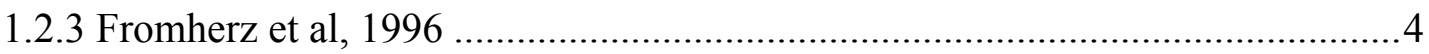

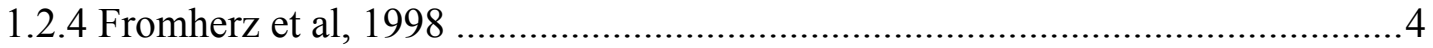

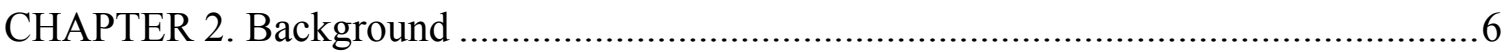

2.1 Nervous System.....................................................6

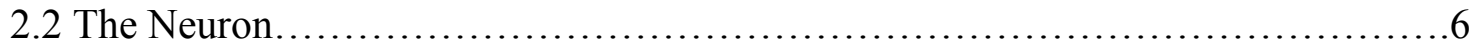

2.2.1 Neuron Structure …..............................................................................

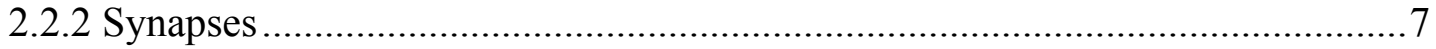

2.2.3 Cell Membrane.......................................................................................

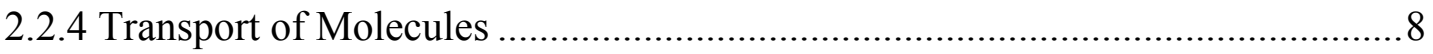

2.2.5 Resting Membrane Potential .........................................................................

2.3 Voltage Gated Ion Channels.......................................... 11

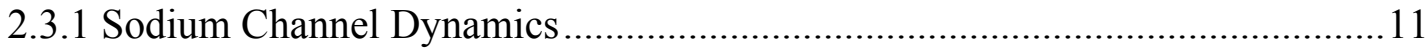

2.3.2 Potassium Channel Dynamics ......................................................................... 11

2.4 Action Potential...................................................... 12 
2.5 Transistors.

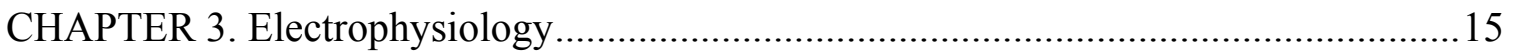

3.1 Recording Techniques................................................ 15

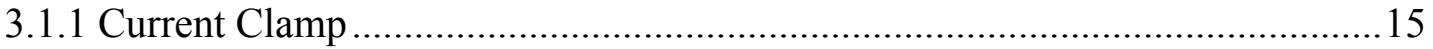

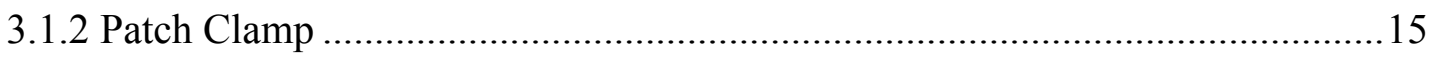

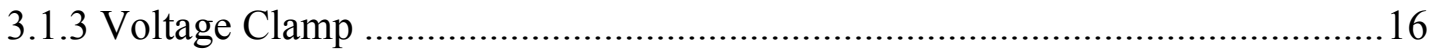

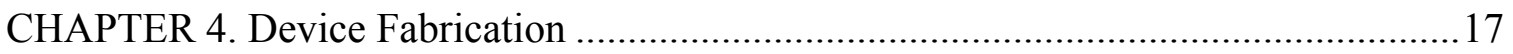

4.1 Transistor Fabrication............................................... 17

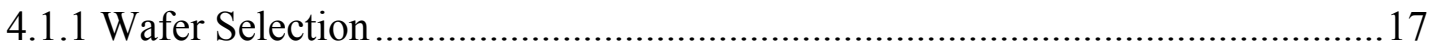

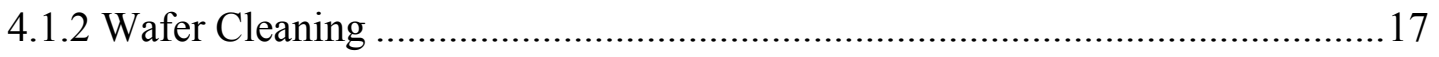

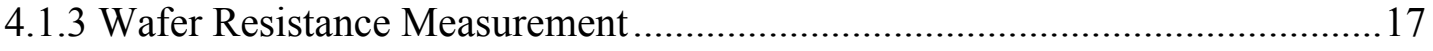

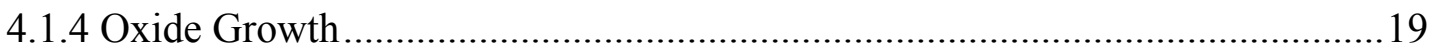

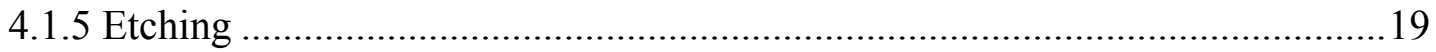

4.1.6 Oxide Removal.......................................................................................... 19

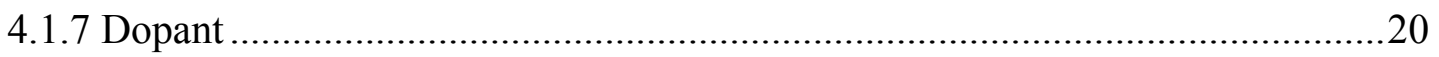

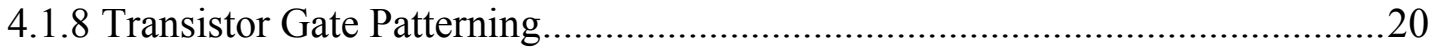

4.1.9 Aluminum Deposition and Patterning ......................................................20

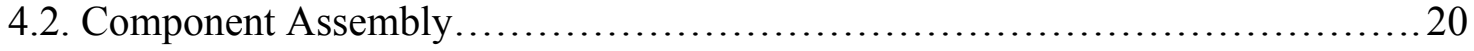

4.2.1 Electrical Insulation and Contacts........................................................20

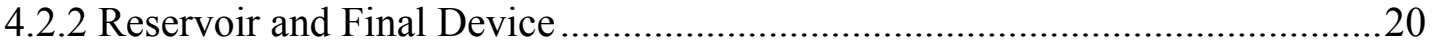

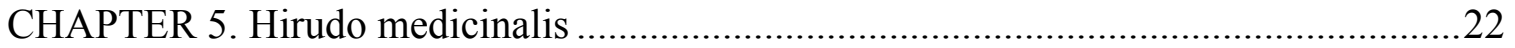

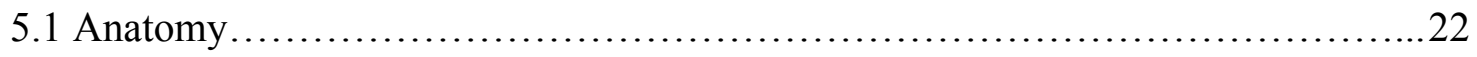

5.2 Leech Care.............................................................. 24 
CHAPTER 6. Methods.

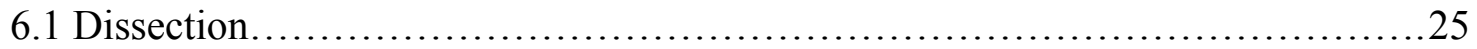

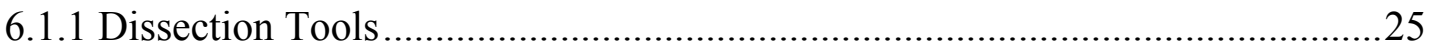

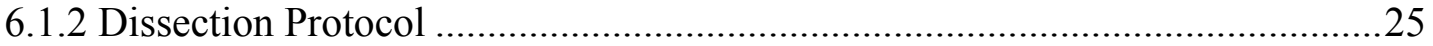

6.2 Neuron Treatment.................................................... 28

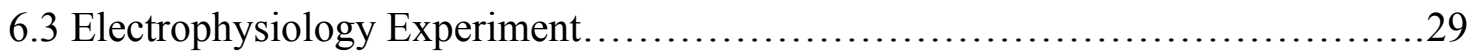

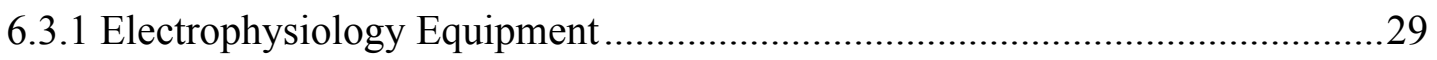

6.3.2 Electrophysiology and Transistor Recording Protocol ..................................30

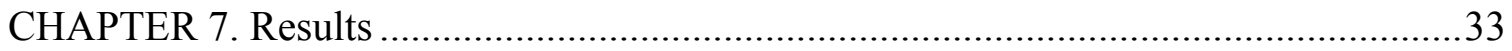

7.1 Oscilloscope Recording Results.......................................33

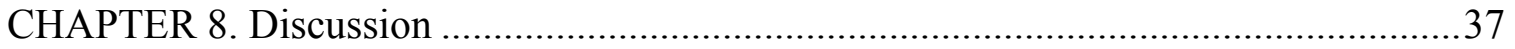

8.1 Project Challenges...................................................... 37

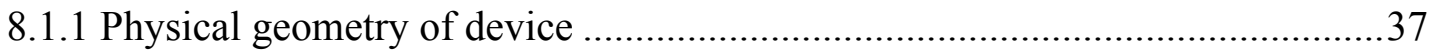

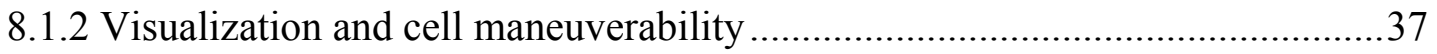

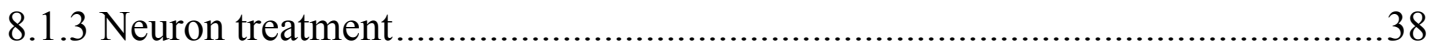

8.2 Concluding Remarks.................................................. 38

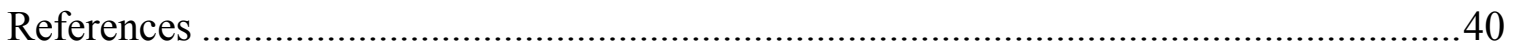

\section{APPENDICES}

Appendix A: Pulled Glass Micropipette Recipes .....................................................42

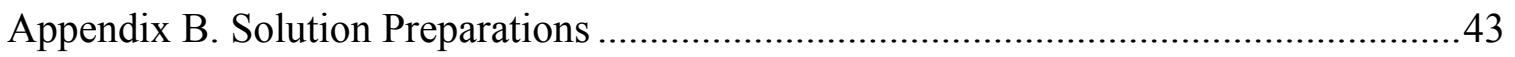

Neuron Culture Solution................................................. 43

Isotonic Leech Bath....................................................43 
Appendix D. Matlab code modeling gating variable and transmembrane potential

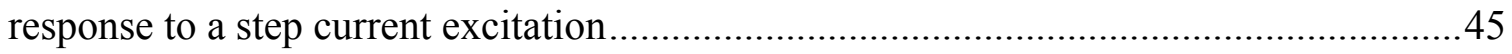

Main Program ........................................................... 45

User-Defined Functions used in Main Program.................................. 48 


\section{LIST OF TABLES}

Page

Table 1. Measured Resistance of Silicon Wafers ..................................................... 18

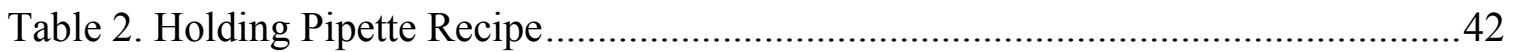

Table 3. Intracellular Microelectrode Pipette Recipe .................................................42 


\section{LIST OF FIGURES}

Page

Figure 1. Diagram of Fromherz's original experimental design with some equivalent

circuit components [1]

Figure 2. Voltage reading resulting from single current pulse and the main result that

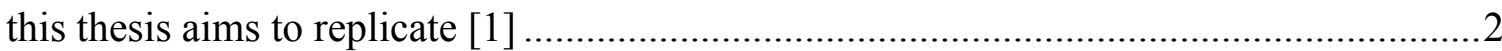

Figure 3. Improved equivalent circuit of the junction between the Retzius cell

membrane and the insulated transistor gate [2]

Figure 4. Electrical trace highlighting the delay between the junction voltage spike

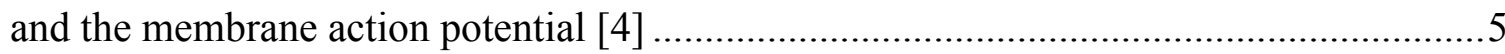

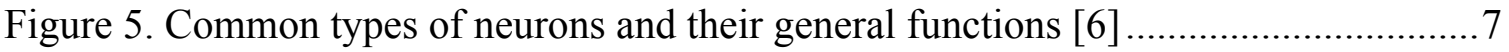

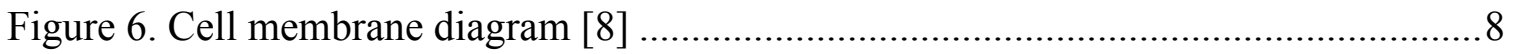

Figure 7. Equivalent circuit representation of the Hodgkin-Huxley model [9]................10

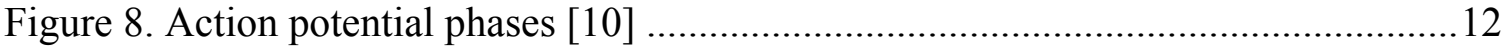

Figure 9. Top: Hodgkin Huxley gating variables (m, n, and h) response to a step

current excitation; Bottom: Corresponding change in transmembrane voltage in

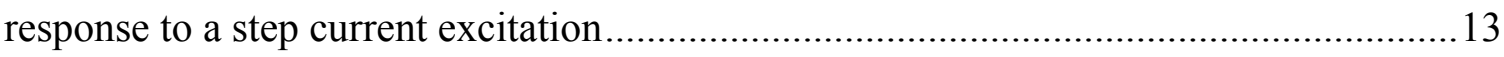

Figure 10. MOFSET terminals cross-section: S, G, D, and B denote source, gate,

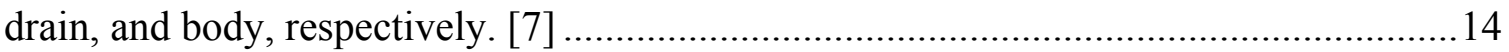

Figure 11. Patch clamp experimental setup [5] .................................................. 15

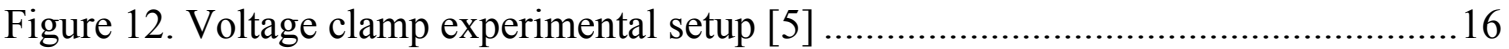

Figure 13. Dopant concentration as a function of wafer resistivity .................................18

Figure 14. Acrylic housing with silicon chip in the center reservoir ............................21

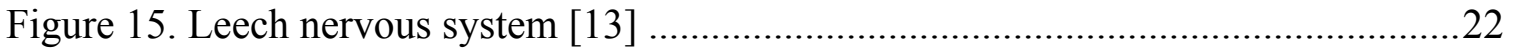


Figure 16. Top: Leech ganglion under microscope; R denotes Retzius cells.

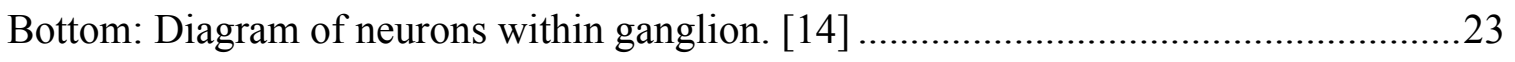

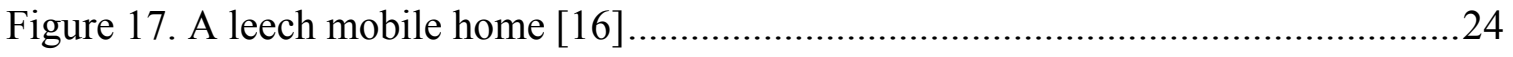

Figure 18. Pinned-out leech with segmental ganglia exposed ....................................26

Figure 19. Ganglion after clearing away black, fibrous blood sinus and connective

tissue

Figure 20. A single neuron aspirated by a micropipette [17] .....................................29

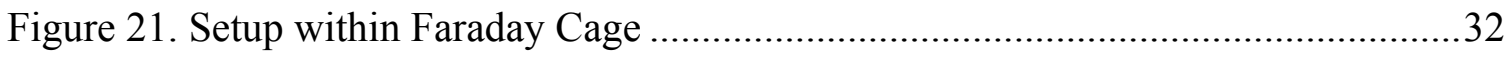

Figure 22. Electrical reading after stimulus. The yellow trace and blue trace

represent the transmembrane and transistor voltage, respectively

Figure 23. Oscilloscope traces resulting from $0.3, .75,1.5$, and $3 \mathrm{nA}$ stimuli, respectively. V1 represents the change in membrane potential in milliVolts that results from the current injected into the Retzius cell; V_trans represents the change in transistor voltage resulting from the change in membrane potential

Figure 24. Electrical reading after 3nA stimulus; cell membrane potential on top and transistor voltage on bottom .36

Figure 25. Correct technique for breaking the holding pipette tip [18] 42 
CHAPTER 1. Introduction

Neural information is transmitted in nervous tissue by the propagation of electrical signals along electrogenic cells. These electrical signals are called action potentials and all involve depolarizing and repolarizing events that occur in the cell [19]. This thesis aims to demonstrate the possibility of studying neuro-electric junctions on the level of a single neuron interfaced with a transistor.

\subsection{Motivation}

The study of a single neuron's electrical behavior in vitro provides a basis of understanding for the further development of neural implants as well as cultured neural networks. Since in vitro neural networks are typically cultured on microelectrode arrays, it is advantageous to study a singular neuro-electronic junction such as the one presented in this thesis.

Additional motivations for selecting to replicate Fromherz's 1991 experiment were that it met an appropriate scope for a Master's thesis and was aligned with the academic interests and skills of the involved students. Furthermore, replication reduces variability in results and adds to the body of work in any field of research. Thus, there is academic merit to reproducing past experiments and validating previous results.

\subsection{Previous Work}

\subsubsection{Fromherz et al, 1991}

This thesis aims to replicate Fromherz's 1991 experimental setup and results [1]. A leech Retzius cell was isolated from a Hirudo medicinalis ganglion using enzymatic treatment and manipulation by pulled glass micropipette. The neuron, denoted by the 
letter $\mathrm{N}$ in Figure 1, was mounted on the insulated gate of a field-effect transistor (FET) and impaled by a sharp microelectrode (ME).

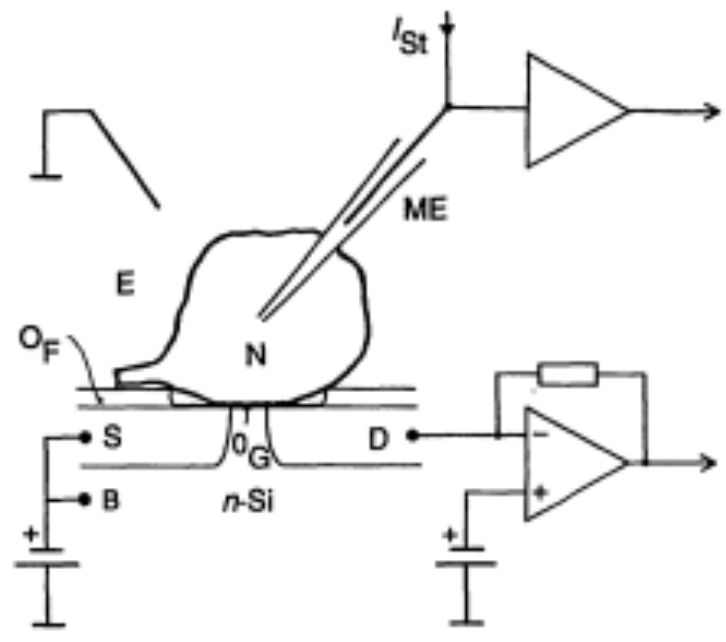

Figure 1. Diagram of Fromherz's original experimental design with some equivalent circuit components [1]

Current was injected into the neuron via the ME to excite the cell, which corresponds to the lower trace in Figure 2. The change in membrane potential affected a change in the field-effect transistor and is shown in the upper trace in Figure 2. Membrane potential measurements were taken on 54 neuron-Si contacts and results found a range of strong, weak, and zero coupling of action potentials with gate voltage.

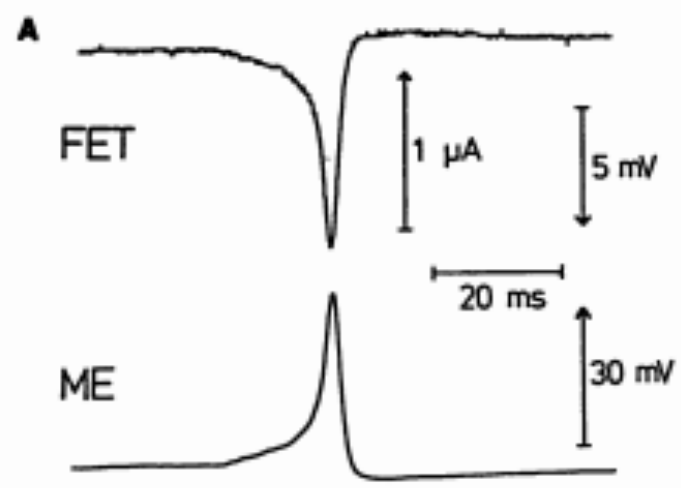

Figure 2. Voltage reading resulting from single current pulse and the main result that this thesis aims to replicate [1] 
The paper suggested that variable coupling might be a result of varying seal strengths between the neuron membrane and the gate. Variable neuron activity could have also contributed to the range of results, as some neurons may have been damaged over the course of the enzymatic treatment and experimental procedure.

\subsubsection{Fromherz et al, 1993}

Fromherz's 1993 work [2] built on his 1991 experiment. While the same FET fabrication and Retzius cell preparation protocol were used, the patch-clamp technique to control the transmembrane potential was now applied, which was a departure from the sharp electrode stimulation in the 1991 experiment. The patch-clamp technique allowed them to study spontaneous activity of the neurons and their spectral responses, as well as develop a detailed equivalent circuit of the junction.

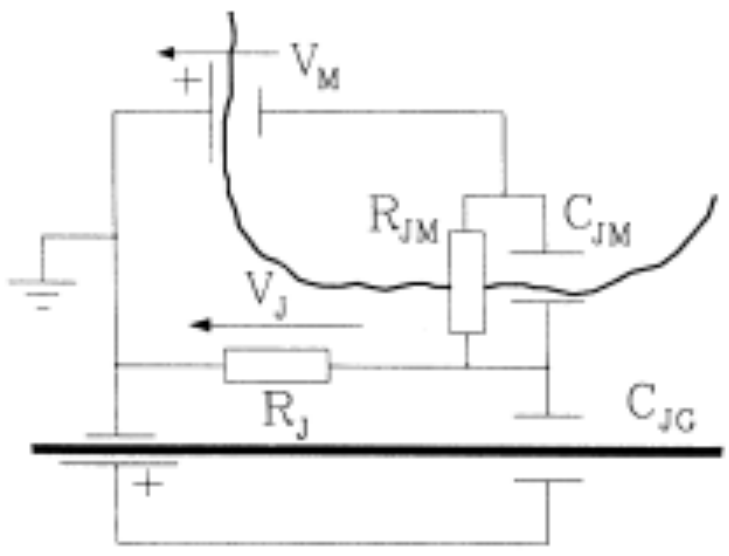

Figure 3. Improved equivalent circuit of the junction between the Retzius cell membrane and the insulated transistor gate [2]

The most relevant finding from the 1993 experiment in terms of this thesis was that the FET response was a result of local coupling of the neuron and silicon, not a change in the potential of the solution surrounding the neuron. 


\subsubsection{Fromherz et al, 1996}

Fromherz's 1996 work [3] quantified neuron-to-silicon adhesion, which determines the strength of electrical signals from the silicon. As in his prior experiments, a neuron was placed on the open metal-free gate of a FET within an oxidized surface of silicon. The patch clamp technique was used to control the intracellular potential. AC voltage stimuli were applied in a frequency sweep from 1 to $15000 \mathrm{~Hz}$. Measurements of the voltage and current at the head of the patch clamp pipette allowed calculations of the attached neuron's impedance. A profile of voltage transfer was established based on measurement of the source-drain current modulations. Further demonstration of the importance of cell adhesion came from the development of an attachment site model using the cable theory.

\subsubsection{Fromherz et al, 1998}

Fromherz's 1998 work [4] involved a more sophisticated technique for mounting neurons onto FETs; the axon stump was attached to the open gate using concanavalin A, a lectin commonly used in cell biology for adhesion. This new methodology led to the discovery of new neuro-silicon junction patterns. In previous studies, the transistor and microelectrode spikes occurred simultaneously after neuron stimulation. This study revealed a biphasic response, with the neuro-silicon junction voltage (as measured by the FET) producing a leading negative peak before the action potential produces a delayed positive peak in the cell membrane. 

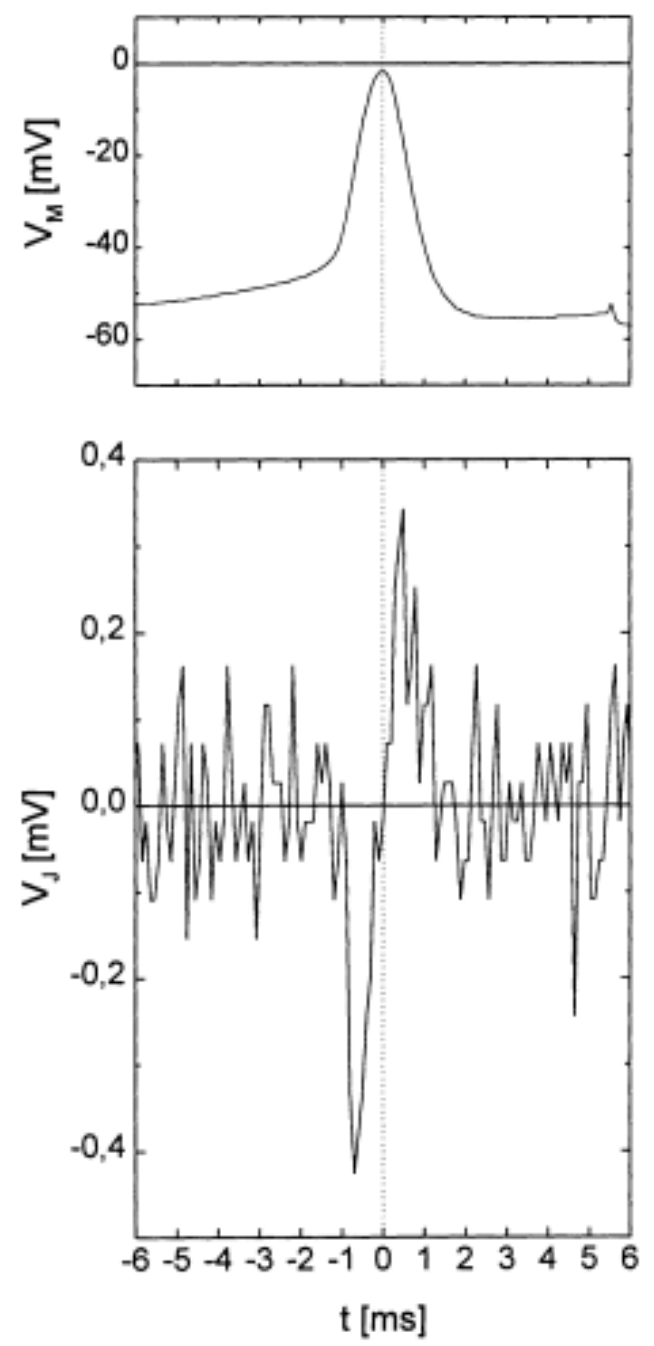

Figure 4. Electrical trace highlighting the delay between the junction voltage spike and the membrane action potential [4] 
CHAPTER 2. Background

\subsection{Nervous System}

The nervous system consists of a network of electrically active cells and tissues. The network can be divided into two parts, the central nervous system (brain and spinal cord) and the peripheral nervous system (everything else). Its primary functions are to received sensory information, process that information, and transmit resultant signals [19]. The success of these functions determines organism survival.

\subsection{The Neuron}

The goal of this section is to understand the neuron in its in vivo environment before attempting to understand its behavior in an in vitro environment.

\subsubsection{Neuron Structure}

It is important to explain neuron structure before discussing neuron function. The soma is the cell body, which contains typical eukaryotic organelles. Axon and dendrites branch off from soma; dendrites typically receive signals, whereas axons typically send

them [19]. A few different types of neurons (shown in Figure 5) are found in the nervous system and as is typical in many systems, their form is specific to their function. 


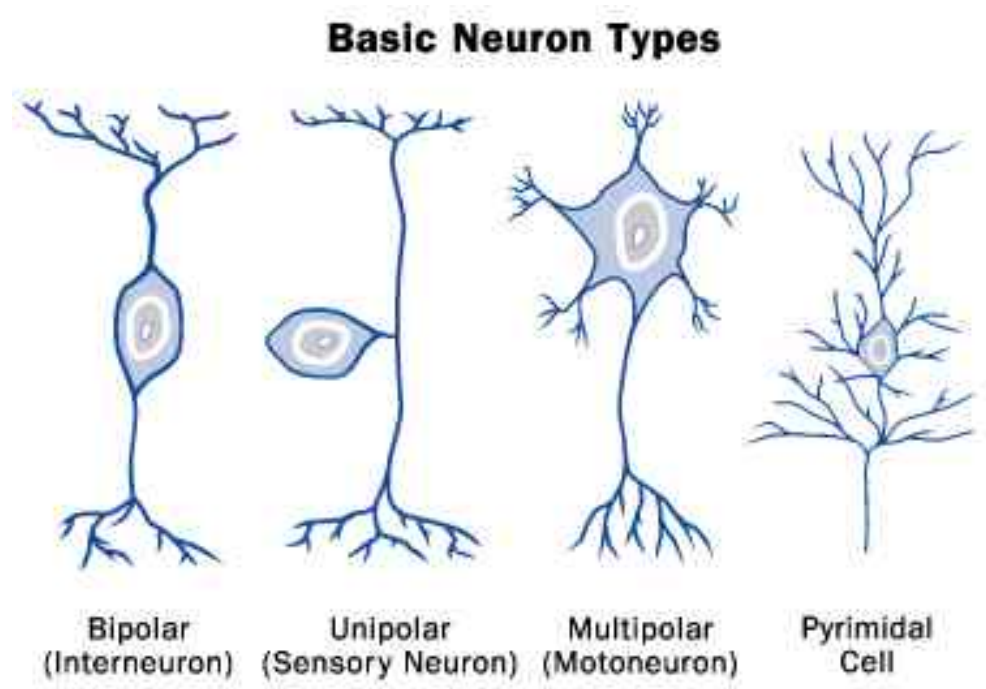

Figure 5. Common types of neurons and their general functions [6]

\subsubsection{Synapses}

The synapse is the location where signals from one neuron are transmitted to another. The action potential in the presynaptic neuron causes the release of neurotransmitter from the synaptic terminal. The neurotransmitter then crosses the synaptic cleft and binds to a receptor on the postsynaptic neuron. Bound neurotransmitter results in the depolarization of the postsynaptic neuron's membrane and the propagation of the action potential (in the postsynaptic neuron) [19]. In the central nervous system, excitatory inputs from a large number of pre-synaptic neurons are required to generate an action potential in a postsynaptic cell.

\subsubsection{Cell Membrane}

The neuronal cell membrane consists of a phospholipid bilayer and maintains the separation of intracellular and extracellular fluids. It is important to note that extracellular fluid is higher in sodium ion concentration than it is in potassium ion concentration, whereas the intracellular fluid is the opposite: lower in sodium ion concentration than it is 
in potassium ion concentration. The hydrophobic "tails" of the phospholipid orient towards themselves; the hydrophilic "heads" of the lipids face outwards, in contact with the surrounding fluids. Within the cell membrane are a variety of embedded transport proteins that further aid in this separation [19]. The next section will discuss how these transport proteins function.

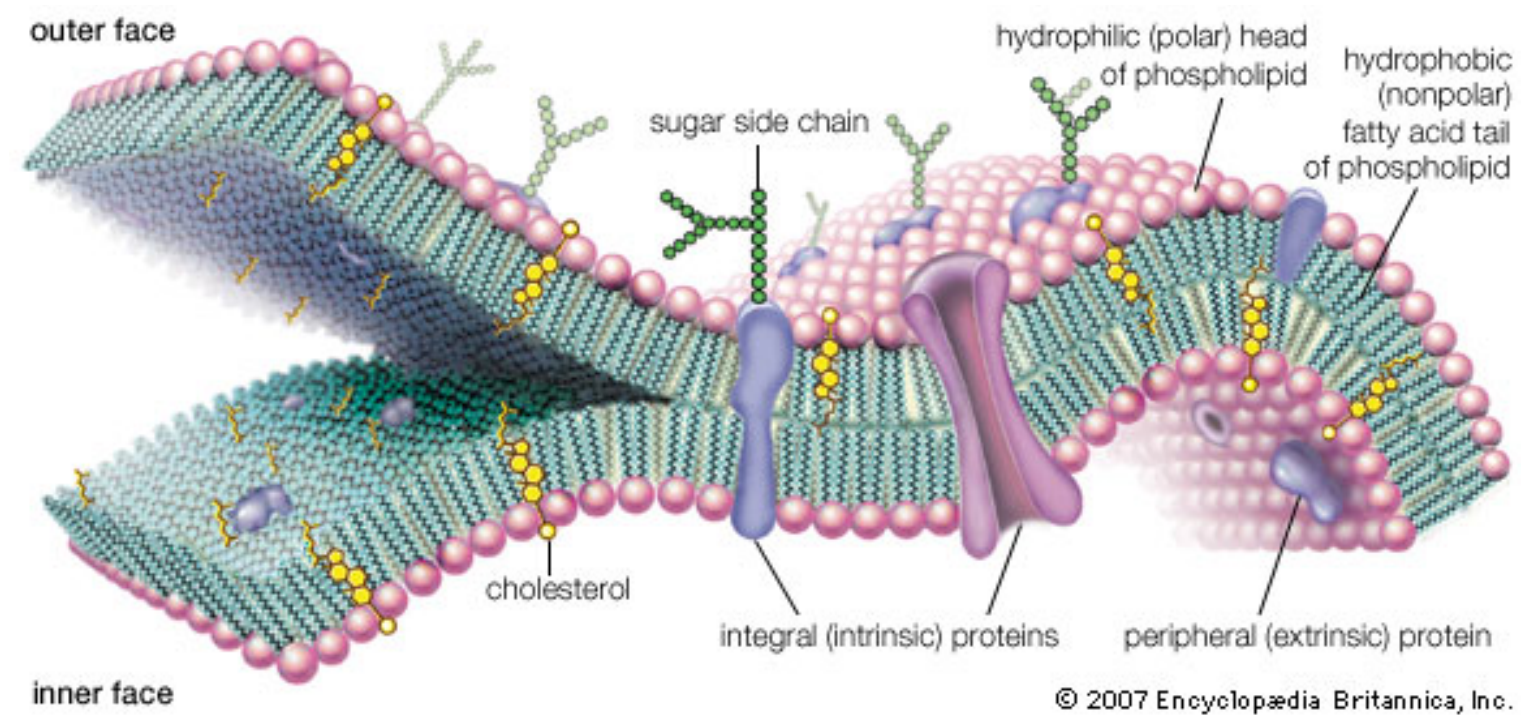

Figure 6. Cell membrane diagram [8]

\subsubsection{Transport of Molecules}

Molecules cross the cell membrane via three general mechanisms: simple diffusion, passive transport, and active transport. Small, hydrophobic molecules are able to diffuse directly through the membrane without the assistance of membrane proteins. Passive transport occurs when ions cross the membrane and travel down their concentration gradient through channel proteins. Ions travelling up their concentration gradient require active transport to cross the cell membrane. Active transport involves adenosine triphosphate (ATP, a source of energy) and molecule-specific carrier proteins. 


\subsubsection{Resting Membrane Potential}

Resting membrane potential is determined by the ionic concentrations of sodium, chloride, and potassium ions and the permeability of the cell membrane to each.

\subsubsection{Nernst Potentials}

The Nernst Potential equation represents the equilibrium potential due to different concentrations of a single ion species on either side of a permeable membrane.

$$
\text { Equation 1. } E_{N}=\frac{R T}{z F} \ln \left(\frac{[N]_{o}}{[N]_{i}}\right)
$$

At room temperature for a mammalian motor neuron, Equation 1 can be rewritten as

$$
\text { Equation 2. } E=\left(\frac{58 m V}{Z}\right) \log _{10}\left(\frac{[N]_{o}}{[N]_{i}}\right)
$$

\subsubsection{Goldman Equation}

The Goldman Equation builds on the Nernst Potential equation by incorporating multiple ion species and steady state transmembrane potential:

$$
\text { Equation 3. } E_{m}=58 m V \log _{10}\left(\frac{\left[\mathrm{K}^{+}\right]_{o}+b\left[\mathrm{Na}^{+}\right]_{o}+c\left[\mathrm{Cl}^{-}\right]_{i}}{\left[\mathrm{~K}^{+}\right]_{i}+b\left[\mathrm{Na}^{+}\right]_{i}+c\left[\mathrm{Cl}^{-}\right]_{o}}\right)
$$

where $o$ denotes ionic concentrations outside the cell, $i$ denotes ionic concentrations inside the cell, and

$$
b=\frac{p_{N a}}{p_{K}} \text { and } c=\frac{p_{C l}}{p_{K}}
$$

where $p$ is the permeability of the membrane to a specific ion. $\mathrm{Na}, \mathrm{Cl}$, and $\mathrm{K}$ represent sodium, chloride, and potassium, respectively. 


\subsubsection{Hodgkin-Huxley Model}

Most, if not all, models of these electrical signals are based on the HodgkinHuxley model. The Hodgkin-Huxley model which is an equivalent circuit model representing the electrical behavior of a neuron membrane as picture below in Figure 7, was developed in 1952 from the results of experiments on giant squid axons.

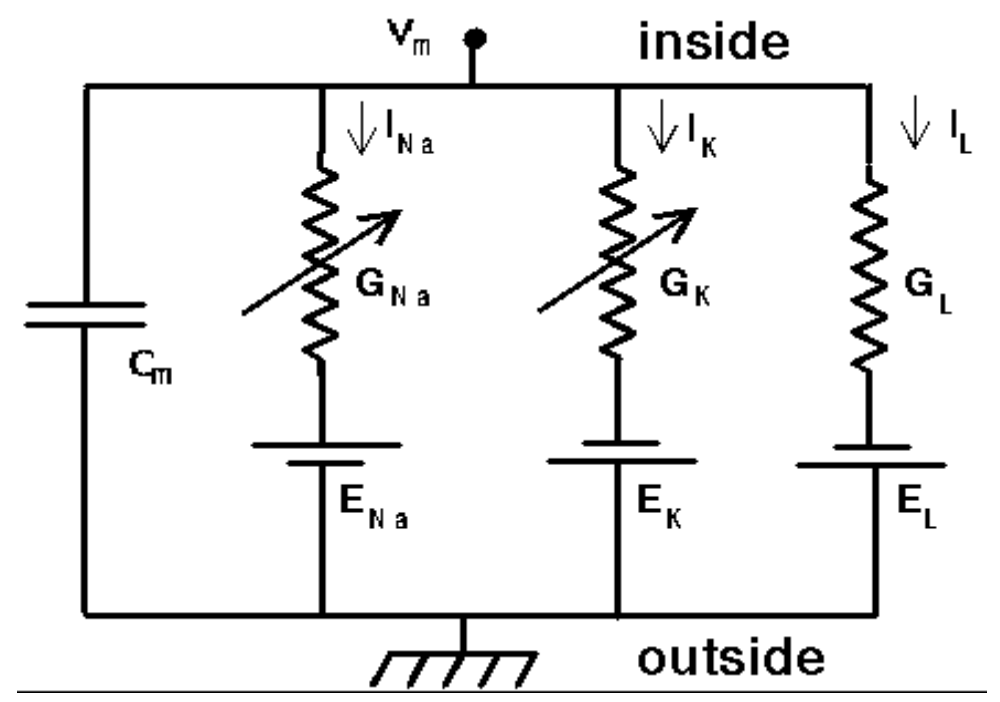

Figure 7. Equivalent circuit representation of the Hodgkin-Huxley model [9]

The governing equation for the model of the neuron can be derived using basic circuit analysis techniques and is as follows:

$$
\text { Equation 4. } I_{m e m}=C_{m e m} \frac{d V_{m e m}}{d t}+\sum_{i} I_{i}
$$

where $I_{m e m}$ is the neuronal membrane total current, $C_{m e m}$ is the membrane capacitance with an assumed value of $1 \mu \mathrm{F} / \mathrm{cm}^{2}, V_{\text {mem }}$ is the transmembrane electrical potential, and $I_{i}$ is the current through the membrane due to the flow of each ion $i$ through the membrane (sodium, potassium, and ions). The equation for $I_{i}$ is given by:

$$
\text { Equation 5. } I_{i}=\alpha \bar{g}_{i}\left(V_{m e m}-E_{i}\right)
$$


where $\alpha$ is a dimensionless ion channel gating parameter, $\bar{g}_{i}$ is the channel maximum conductance of the ion, and $E_{i}$ is the ion's equilibrium potential.

\subsection{Voltage Gated Ion Channels}

Channel proteins involved in active transport have two states: open, in which ions can move through the protein, and closed, in which ions cannot. In the case of voltage gated ion channels, changes in membrane potential and time determine the state of the channels. Depolarization increases the likelihood that channels will be open; hyperpolarization decreases that likelihood. This is called the gating particle, a positively charged particle that affects the conformation and resulting conductance of the voltage gated ion channels.

\subsubsection{Sodium Channel Dynamics}

Hodgkin and Huxley experimentally determined that activation gates (known as $\mathrm{m}$ gates) and inactivation gates (h gates) affect the sodium channels. At resting membrane potential, voltage dependent sodium channels are in a closed state. The sodium channel's conductance increases as a result of the activation gates opening due to an increase in membrane potential. The inactivation gates bind the gating particle a few milliseconds after the activation gates open and sodium ion influx ceases.

\subsubsection{Potassium Channel Dynamics}

Voltage dependent potassium channels only have activation gates, the $\mathrm{n}$ gates. At resting membrane potential, this gate is closed. When membrane potential increases during depolarization, the activation gate binds the gating particle after a delay of a few 
milliseconds. The opening of the potassium channel results in potassium ions moving down their concentration gradient to the extracellular side of the membrane.

\subsection{Action Potential}

Action potentials are electrical signals that transmit information in nervous tissue. An increase in membrane potential triggers action potentials. The reduction can be a depolarization of 10 to $20 \mathrm{mV}$ but it must be large enough to reach threshold level. If the membrane potential does not reach the threshold, the potential decays back to resting baseline. Action potentials are binary occurrences in the sense that they either happen fully or not at all. Once the threshold is met, the action potential goes through the same stages each time: depolarization, repolarization, hyperpolarization, and return to baseline potential.

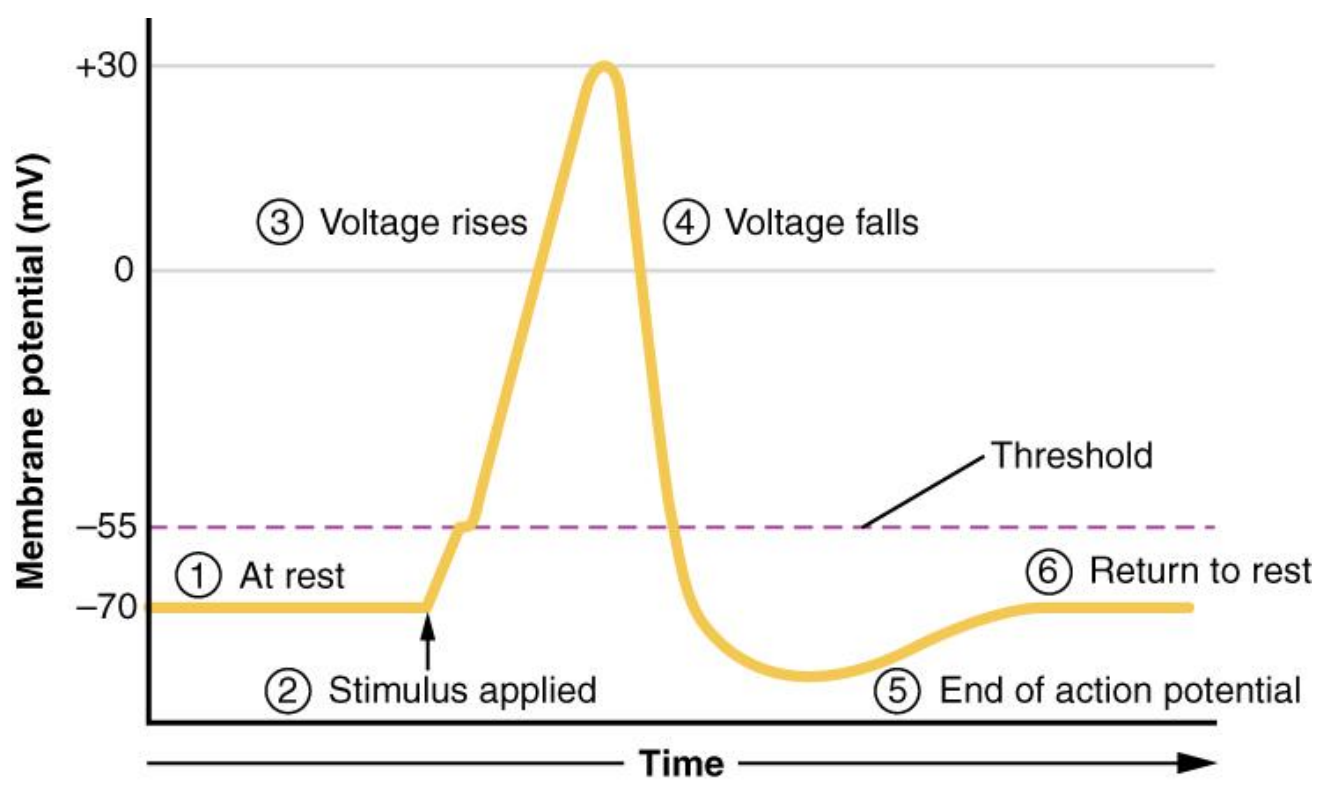

Figure 8. Action potential phases [10]

Each of these phases is associated with specific changes in ionic membrane permeability. During depolarization, the membrane permeability to sodium ions begins to 
increase, resulting in an influx of sodium ions. During repolarization, the membrane permeability to potassium begins to increase, resulting in an efflux of potassium ions.

During hyperpolarization, the membrane potential drops below resting potential during the refractory period, which prevents the immediate firing of another action potential. After the refractory period, membrane potential returns to its resting baseline. A graphical model of this phenomenon is shown below in Figure 9 and the Matlab code used for this simulation can be found in Appendix D.
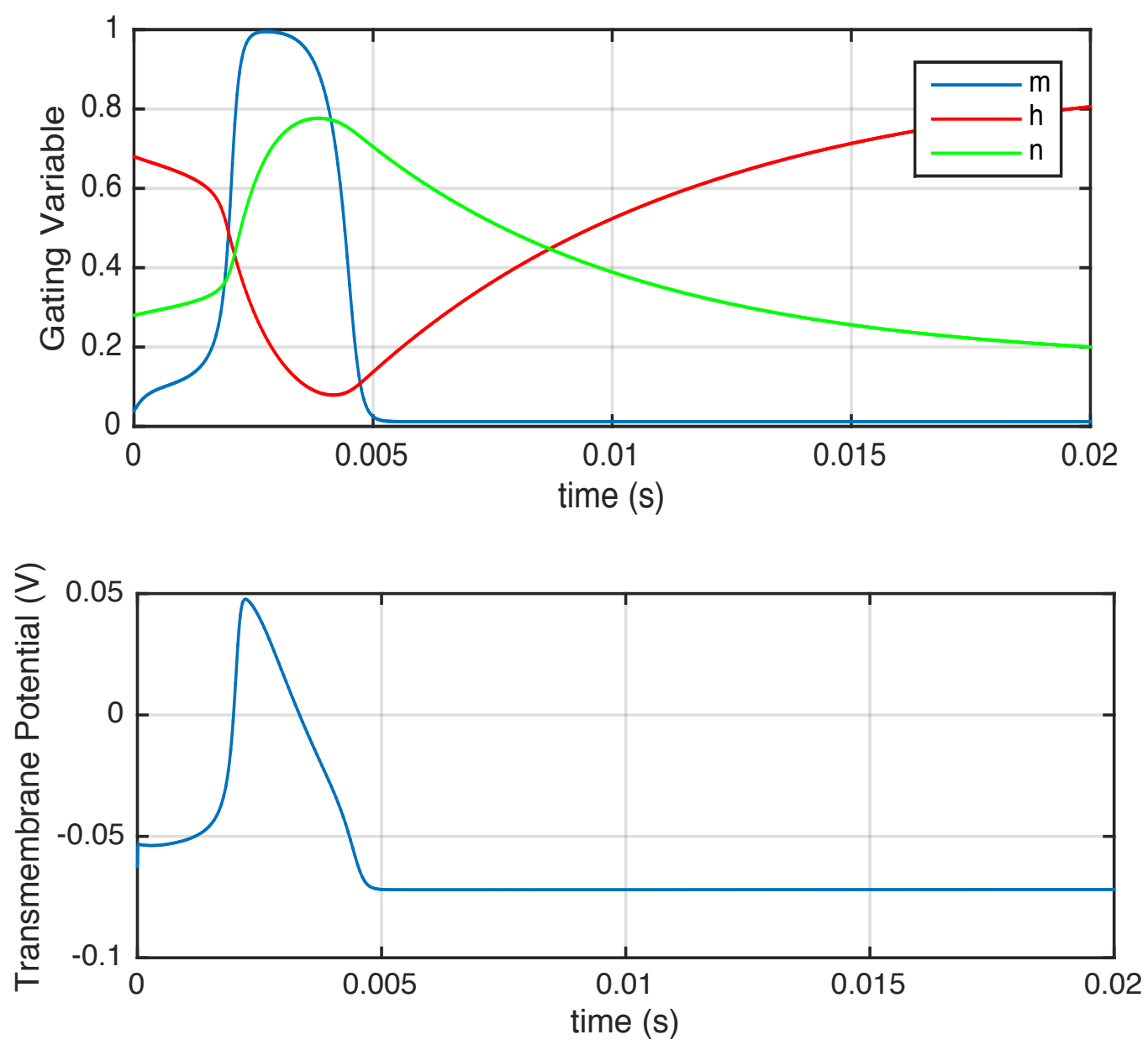

Figure 9. Top: Hodgkin Huxley gating variables (m, n, and h) response to a step current excitation; Bottom: Corresponding change in transmembrane voltage in response to a step current excitation 


\subsection{Transistors}

A transistor is an electronic semiconductor device with three terminals.

Transistors are composed of doped silicon and the dopants affect the conductivity of the silicon. There are two types of doped silicon: N-type (which adds elements with 3 valence electrons to create holes) and P-type (which adds elements with 5 valence electrons to create free charge carriers). Transistors can amplify current signals.

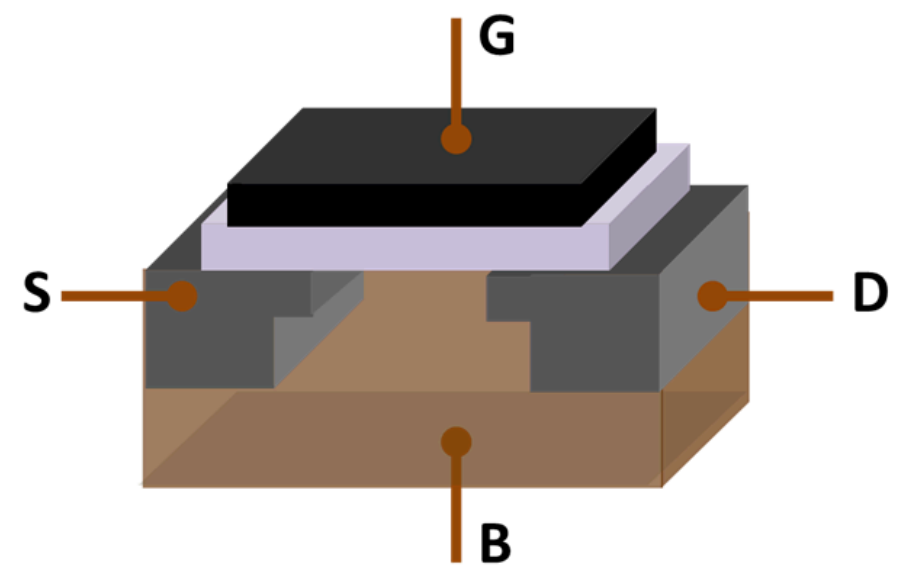

Figure 10. MOFSET terminals cross-section: S, G, D, and B denote source, gate, drain, and body, respectively. [7] 
CHAPTER 3. Electrophysiology

\subsection{Recording Techniques}

\subsubsection{Current Clamp}

The current clamp technique is utilized in this thesis. Current clamp experiments consist of using a microelectrode to inject current into and record resulting changes in transmembrane potential of an impaled cell. This experimental setup allows insight into overall membrane activity.

\subsubsection{Patch Clamp}

The patch clamp technique involves fusing a section of the cell membrane with a microelectrode by applying gentle suction. This experimental technique is particularly advantageous in studying the activity of individual ion channels present in the patch section of the membrane.

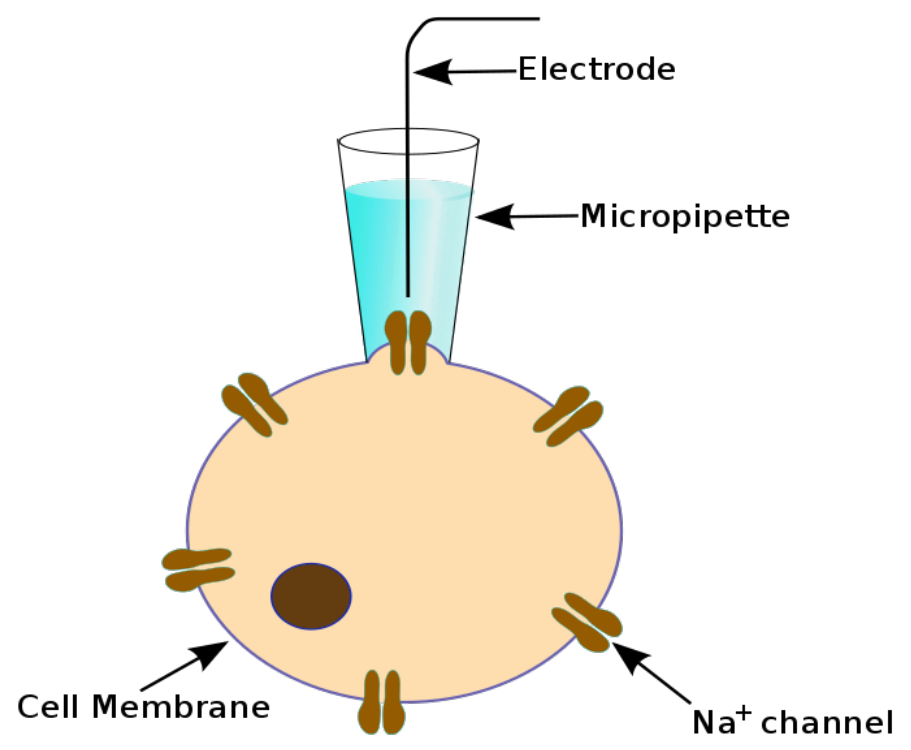

Figure 11. Patch clamp experimental setup [5] 


\subsubsection{Voltage Clamp}

The voltage clamp setup includes a command voltage (denoted as a signal generator in Figure 12), a voltage amplifier, and a current monitor. The intracellular and extracellular electrodes measure the membrane potential, which is compared to the command voltage from the signal generator via a feedback amplifier. The current monitor, another electrode, pushes current into the cell based on this feedback to hold the membrane potential constant at the command voltage. This allows the measurement of transient changes in membrane ionic conductance.

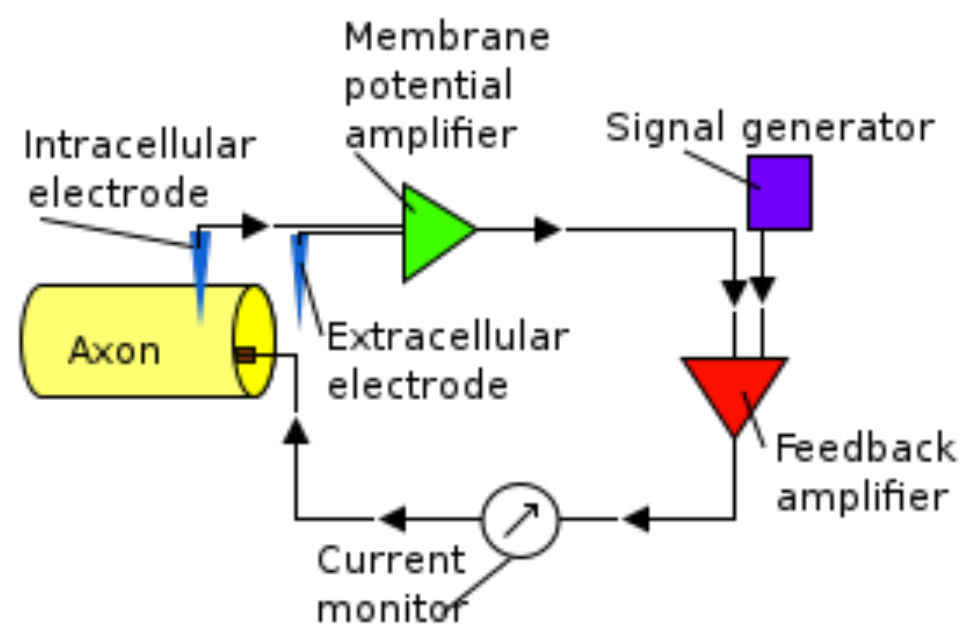

Figure 12. Voltage clamp experimental setup [5] 
CHAPTER 4. Device Fabrication

This chapter presents a brief and simplified overview of the steps taken to fabricate the device in order to facilitate understanding of this thesis as a whole.

\subsection{Transistor Fabrication}

\subsubsection{Wafer Selection}

Both $\mathrm{N}$ type and $\mathrm{P}$ type wafers of $100 \mathrm{~mm}$ diameter were selected for use in this thesis; $100 \mathrm{~mm}$ diameter wafers are the standard used at Cal Poly.

\subsubsection{Wafer Cleaning}

In order to remove organic compounds and silicon oxide from the wafer, the wafer went through three baths: a "piranha bath" of hydrogen peroxide and sulfuric acid, a bath of buffered hydrofluoric acid, and a rinse with deionized water.

\subsubsection{Wafer Resistance Measurement}

A four-point probe measured the surface resistance of the wafers to verify the previously defined specification of 5 to $10 \mathrm{Ohm}-\mathrm{cm}$. Results of these measurements are shown in Table 1. This information allows the calculation of dopant concentration in each wafer by referring to Figure 13. 
Table 1. Measured resistance of silicon wafers

\begin{tabular}{|l|l|l|l|}
\hline Wafer Number & Dopant Type & $\begin{array}{l}\text { Measured } \\
\text { Resistance }(\mathrm{Ohm}- \\
\mathrm{cm})\end{array}$ & $\begin{array}{l}\text { Resistance Spec } \\
(\mathrm{Ohm}-\mathrm{cm})\end{array}$ \\
\hline 1 & $\mathrm{~N}$ & 7.53 & $5-10$ \\
\hline 2 & $\mathrm{~N}$ & 5.34 & $5-10$ \\
\hline 3 & $\mathrm{P}$ & 9.44 & NA \\
\hline 4 & P & 8.47 & NA \\
\hline
\end{tabular}

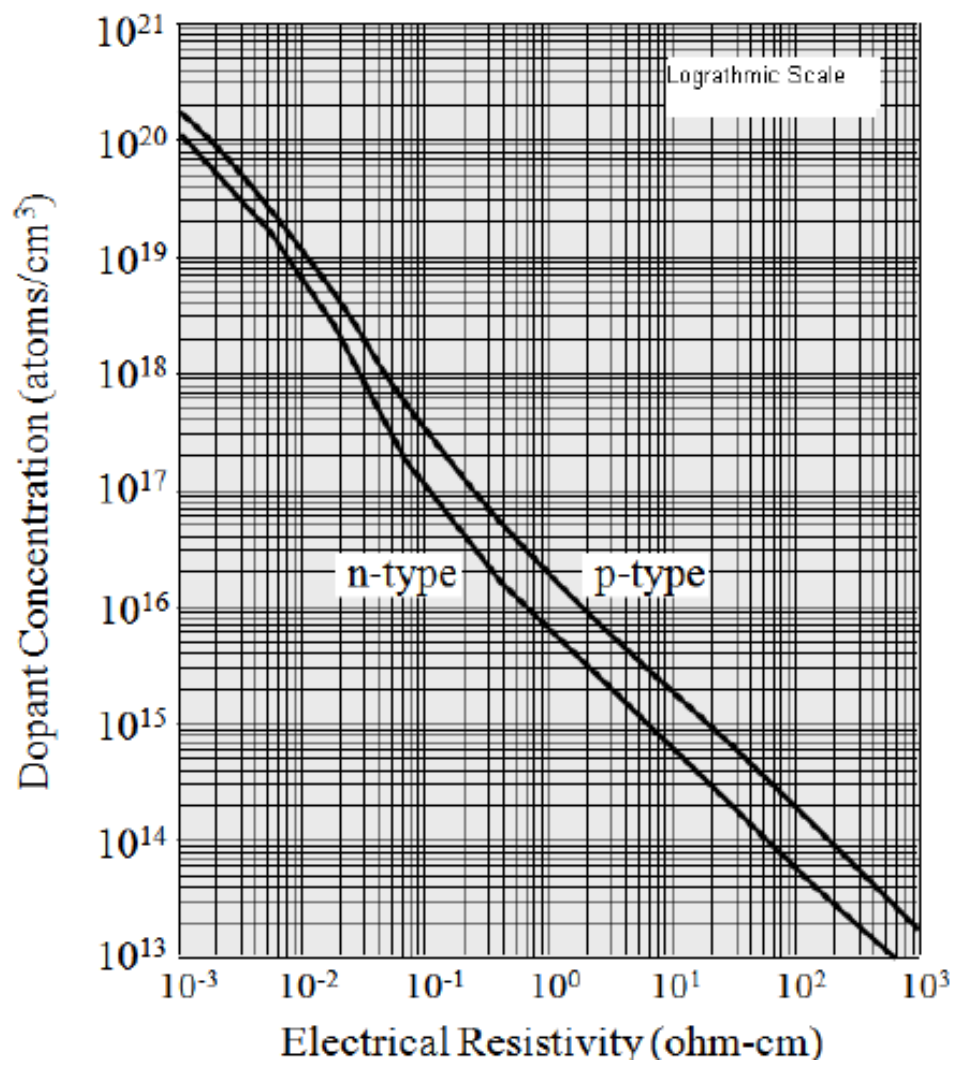

Figure 13. Dopant concentration as a function of wafer resistivity 


\subsubsection{Oxide Growth}

Silicon-oxide masks of 5000 angstroms were grown on the wafers in order to stabilize the wafer surfaces for later thermal diffusion of dopants. In order to determine values for time and temperature parameters, the Deal-Grove model was applied to this process. The Deal-Grove model is based on Henry's law, Fick's first law, and a general first order reaction equation, which describes the oxide growth.

\subsubsection{Etching}

Positive photoresist and primer were spun onto the wafer to form a coating of uniform thickness to prepare the wafer for the etching process. The wafer is then exposed to UV light, which makes the positive photoresist soluble to the chemical developer. A patterned shutter in front of the UV light source accomplishes the selective exposure of wafer to UV light. After exposure to the UV light, the wafer is placed in the chemical developer bath to remove soluble photoresist. The final step in the etching process is cleaning the wafer with deionized water and isopropanol.

\subsubsection{Oxide Removal}

The wafer was submerged in hydrofluoric acid to selectively remove the silicon oxide layer as part of the wet etching process. Timing of this process is meticulous as under- and over-etching affects the size of the wafer features and consequently the performance of the final device. 


\subsubsection{Dopant}

Boron oxide dopant is spun onto etched wafer in a relatively thick layer to allow diffusion of atoms into the wafer. The dopant diffuses into the transistor wells during exposure to high temperature in two steps: pre-dep and drive in.

\subsubsection{Transistor Gate Patterning}

The region of oxide over the transistor gate is too thick for the purposes of this project and some of the layer must be removed to increase the sensitivity of the gate. This removal is accomplished using photoresist and chemical etching.

\subsubsection{Aluminum Deposition and Patterning}

A sputtering process deposits aluminum onto the wafer, which is important for later developing electrical connections to the transistor. The patterning of the aluminum layer is accomplished using photoresist and chemical etching.

\subsection{Component Assembly}

\subsubsection{Electrical Insulation and Contacts}

A negative resist of SU-8 was spun onto the chip to form an electrically isolated layer. After exposure to light, the aluminum contact pads were the only sections of the wafer not insulated. Wires were then attached to the aluminum transistor contact pads via a conductive epoxy.

\subsubsection{Reservoir and Final Device}

After several design iterations, the final device's circular reservoir was laser cut into a piece of acrylic. The wafer chip containing the transistor and electrical traces was glued in using Polydimethyl Siloxane. The wires were covered by silicone and acrylic in 
order to anchor them to the reservoir as well as electrically isolate them. Additionally, Poly-L-Lysine was coated on the gate before each experiment in order to promote cell adhesion, which was an essential parameter in the success of the electrical recordings.

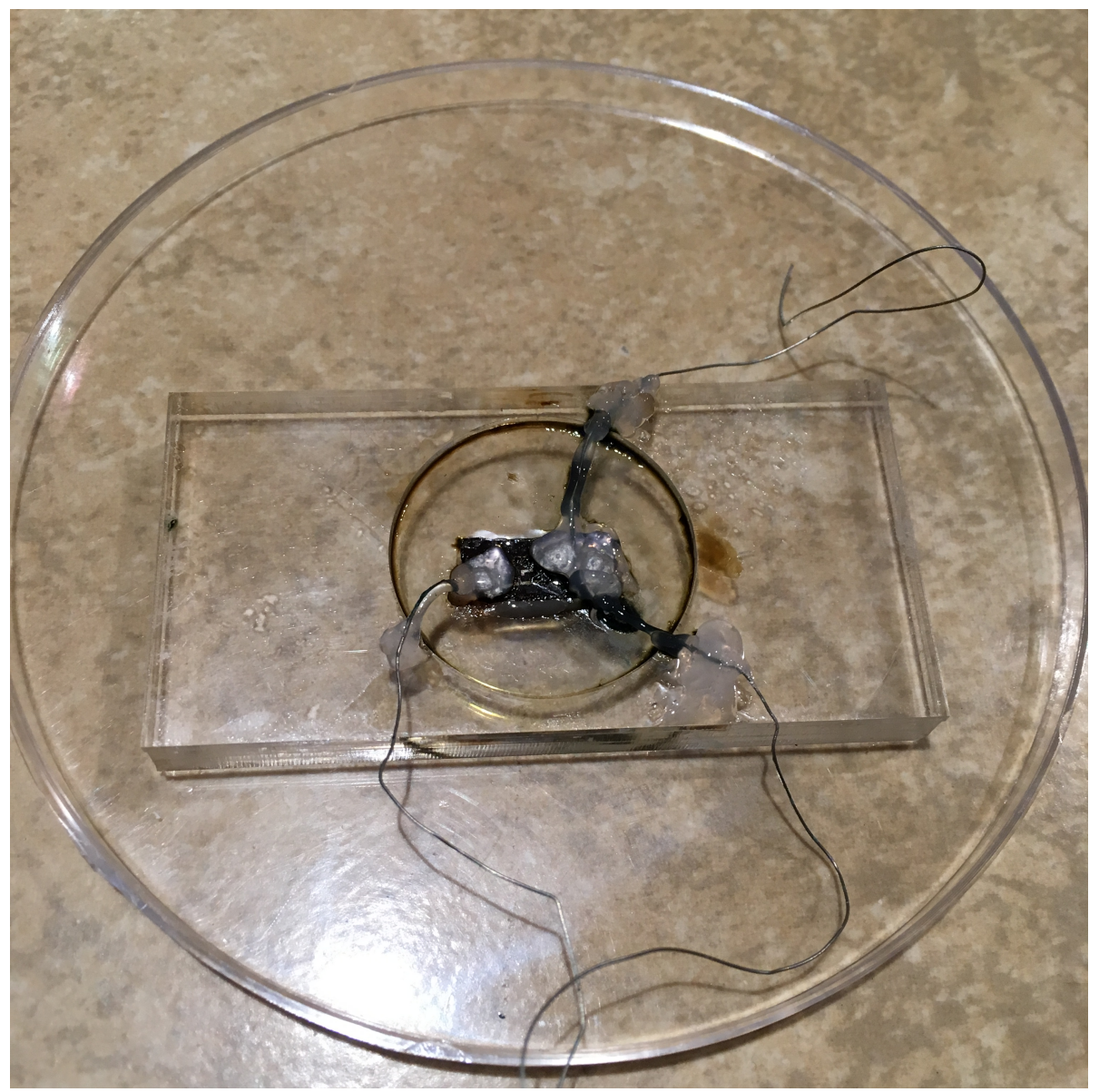

Figure 14. Acrylic housing with silicon chip in the center reservoir 
CHAPTER 5. Hirudo medicinalis

\subsection{Anatomy}

Hirudo medicinalis, commonly known as the medicinal leech, is a hermaphroditic semiaquatic annelid worm and is frequently used as a model organism in neurobiology. The leech central nervous system (pictured in Figure 15) consists of a 'head brain', segmental ganglia, and a 'tail brain'. These ganglia comprise the ventral nerve cord, which is contained within the ventral blood sinus $[12,13]$.
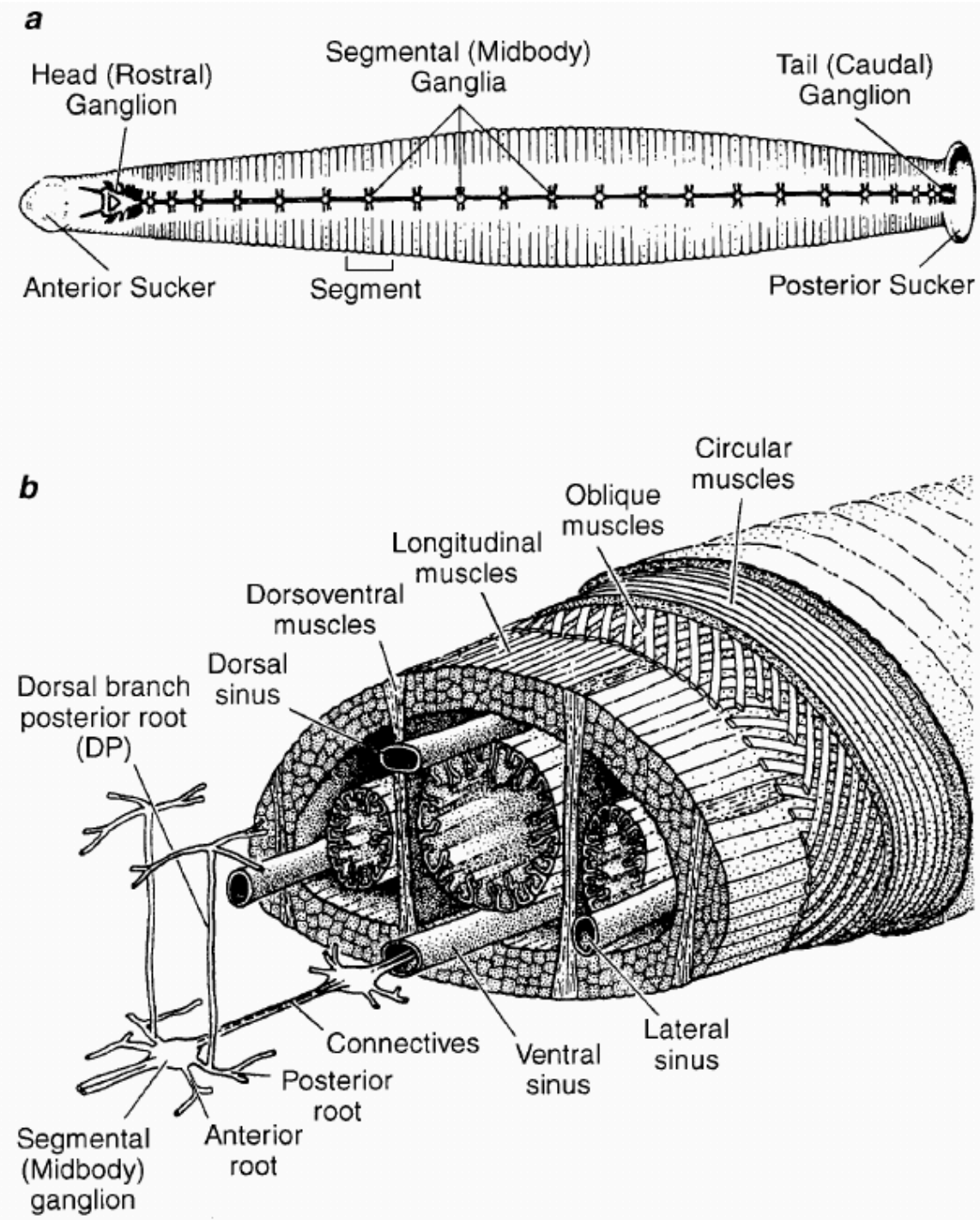

Figure 15. Leech nervous system [13] 
Each leech ganglion contains approximately 400 neurons, ranging from 15-70um in diameter. The arrangement of these neurons within each ganglion (Figure 16) has been established and is consistent from ganglia to ganglia and from leech to leech. This consistency and the relatively large size of the neurons are a couple of the advantages of using Hirudo medicinalis for this thesis.
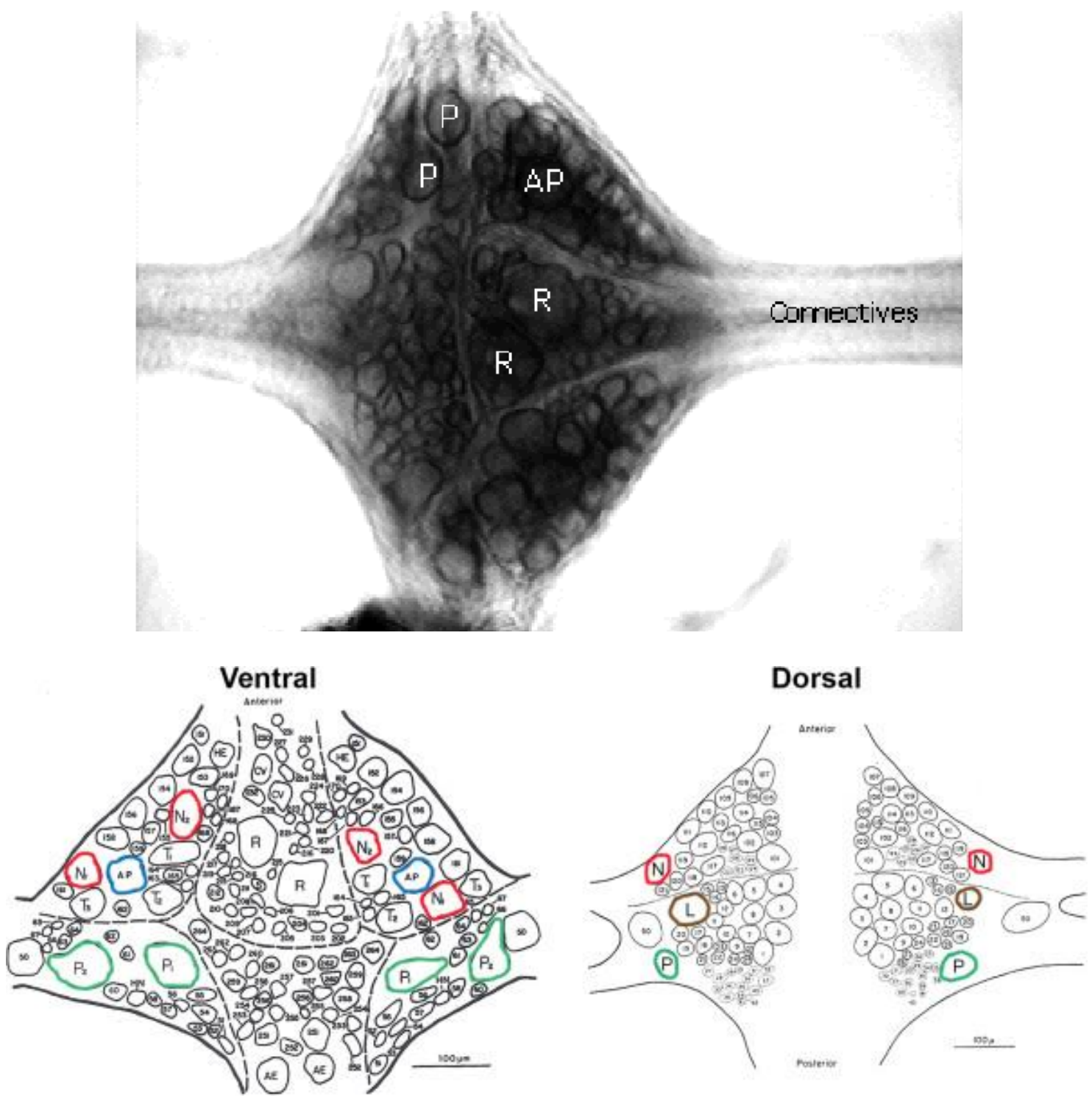

Figure 16. Top: Leech ganglion under microscope; R denotes Retzius cells. Bottom: Diagram of neurons within ganglion. [14] 


\subsection{Leech Care}

Leeches were purchased from Leeches U.S.A. and were placed in the refrigerator upon delivery (still in the bag they had been shipped in). Refrigeration is necessary in order to keep the leeches sedate and easy to work with. While the leeches came down to refrigerator temperature, a gallon of distilled water mixed with 2 grams of HIRUDO Salt was prepared and also brought down to refrigerator temperature. After 24 hours of refrigeration, the leeches were transferred into a plastic container called a 'Leech Mobile Home', filled about $2 / 3$ of the way up with the previously described saltwater. The Mobile Home's lid was replaced and the Mobile Home was returned to the refrigerator. The saltwater needed to be replaced approximately every one to two weeks and the dirty saltwater was disposed of down the drain of a lab sink.

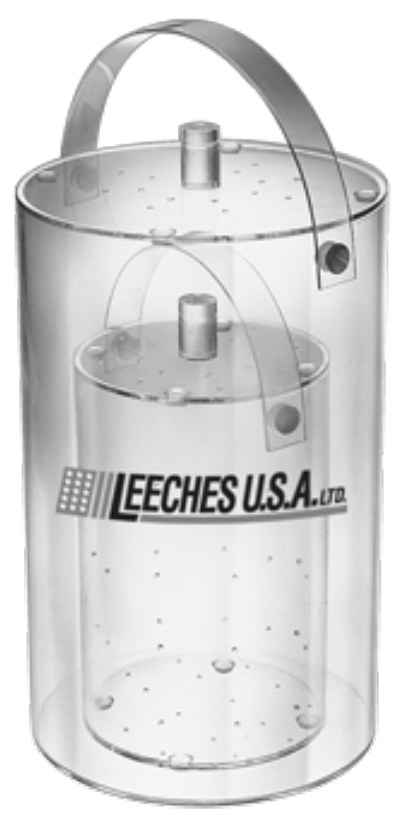

Figure 17. A leech mobile home [16] 
CHAPTER 6. Methods

\subsection{Dissection}

6.1.1 Dissection Tools

1. Large Tongs

2. Ringer's Solution

3. Leeches

4. Leech Mobile Home

5. Light Source

6. Dissecting Microscope

7. Paper Towels

8. Push Pins

9. Surgical Scalpel

10. Microscissors

11. Micro-dissecting forceps

12. Silicone-Filled Petri dish

13. Holding micropipette with tip inner diameter $<60$ um (see Appendix A for further details

\subsubsection{Dissection Protocol}

- Use tongs to remove leech from mobile home and place leech on petri dish 
- Pin one end of the leech close to the side of the dish, dark and patterned side up. Using another pin, impale the other end of the leech, stretch it as far as possible across the dish, then pin it down.

- Use scalpel to make a horizontal cut a quarter of the way up from the centerline of the leech closest to you. The cut should be approximately the middle $2 / 3$ of the leech and be just deep enough to penetrate the exterior skin. Gently pass the scalpel over the incision a few times to ensure the cut is deep enough.

- To maximize visualization of the ganglia, pin both sides of the incision open so the leech's spinal cord is exposed (as shown in Figure 18). You may need to use microscissors at this point to cut away some superficial connective tissue covering the spinal cord.

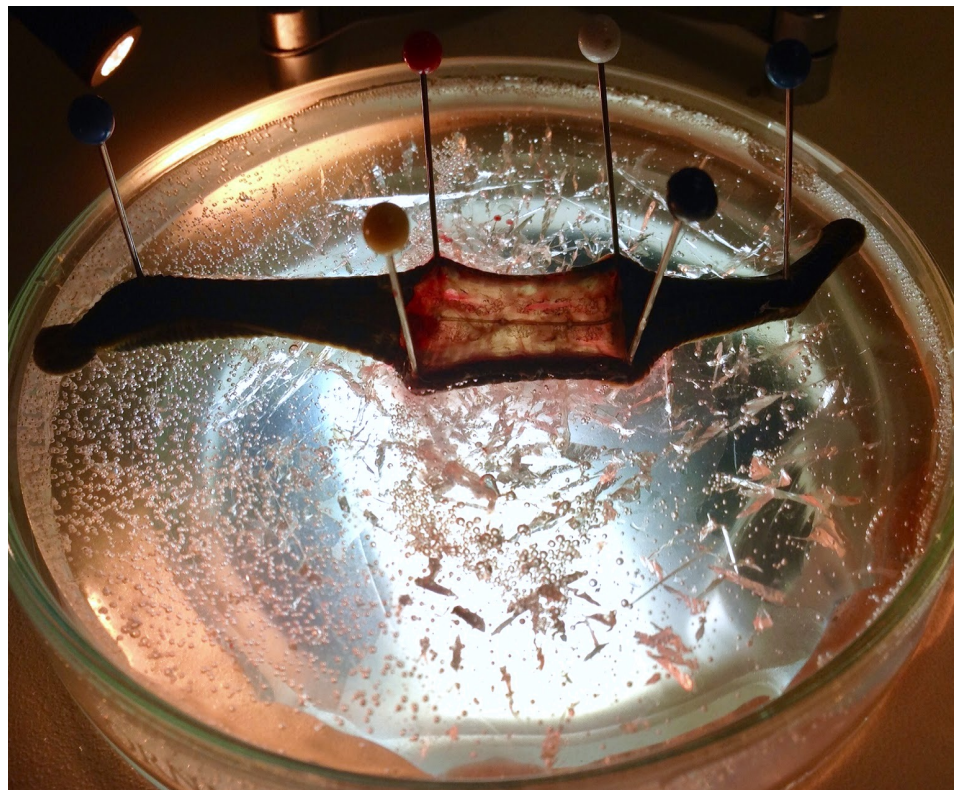

Figure 18. Pinned-out leech with segmental ganglia exposed 
- Focus on one or two ganglia of interest under the microscope using a combination of coarse and fine focus adjustments.

- With micro-dissecting forceps, pull the black fibers off the desired ganglia with small, gentle motions. It is better to pull towards the cell body than away from the cell body as to minimize the risk of prematurely severing the connectives that join adjacent ganglia.

- Once the ganglion has been cleared of surrounding black fibers and other debris (as per Figure 19), use microscissors to cut through the top, bottom, left, and right connectives that anchor the ganglion.

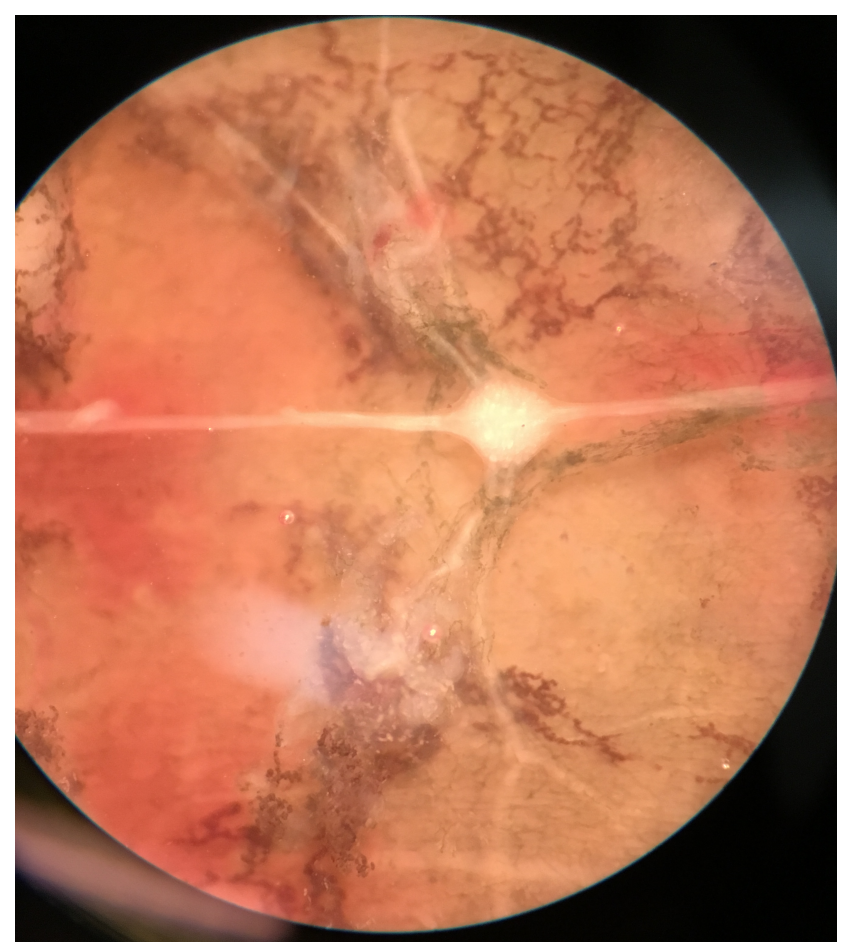

Figure 19. Ganglion after clearing away black, fibrous blood sinus and connective tissue

- Refer to Appendix C for leech carcass disposal protocol. 


\subsection{Neuron Treatment}

- Use micro-dissecting forceps to move detached ganglion into dish containing the silicon chip and collagenase/dispase mixed with L-15 solution.

- Place on shake table for 30 minutes.

- After 30 minutes, use holding micropipettes to isolate one of the two Retzius cells in ganglion. This can be performed under a standard dissection microscope. The Retzius cells are noticeably larger than the rest of the cells contained in the ganglion and should be easy to identify. One method of isolating the Retzius cells is to first rupture the remaining connective tissue that contains the ganglion's neurons, and then to gently maneuver a Retzius cell using the pulled end of the holding pipette. If the pipette has been pulled properly, the cell will loosely suction itself to the end of the pipette without getting fully sucked up into the pipette. 

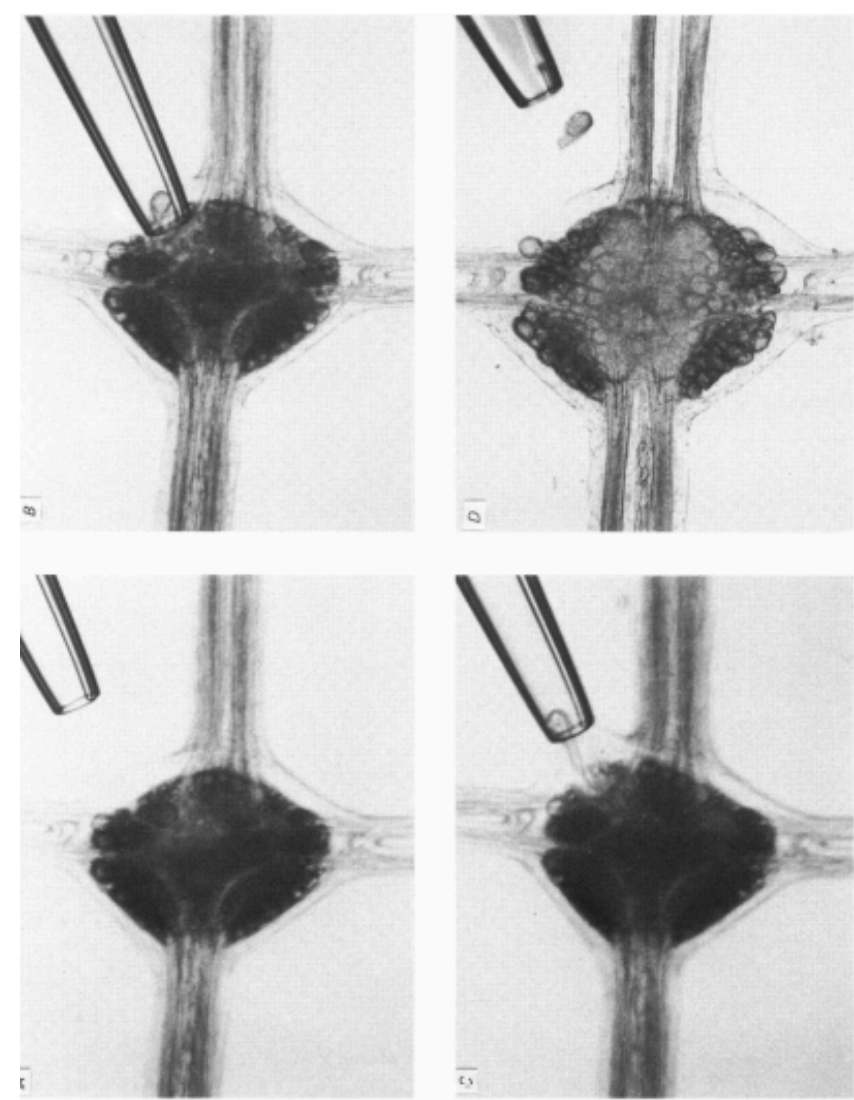

Figure 20. A single neuron aspirated by a micropipette [17]

- Using the holding micropipette, maneuver the aspirated cell from the location of the Retzius cell isolation within the acrylic housing's reservoir into the well just above the transistor gate. It is helpful to use the holding pipette to press the cell securely into the well to increase the strength of the transistormembrane seal but be careful not to compromise the integrity of the cell's membrane.

\subsection{Electrophysiology Experiment}

\subsubsection{Electrophysiology Equipment}

1. Potassium Chloride Solution

2. Axoclamp 900A amplifier 
3. Axoclamp 900A software

4. Computer

5. Dark Field Microscope

6. Digidata 1440a DAQ unit

7. Extracted leech Retzius cell

8. Faraday cage

9. Head stage

10. Light source

11. Microfil

12. Micropipettes

13. Oscilloscope

14. Recording electrodes

15. Ringer's Solution Silver

16. Silver-Chloritized wires

17. Syringe

18. DC power supply

19. Banana clips

20. Alligator clips

\subsubsection{Electrophysiology and Transistor Recording Protocol}

- With as little extraneous motion as possible, transport the dish onto the stage in the Faraday cage.

- Focus the microscope in the Faraday cage on the microelectrode tip 
- Adjust the micromanipulator so that the tip of the sharp electrode is in solution surrounding the transistor gate.

- Set up AxoClamp software and calibrate settings; pipette capacitance, bridge balance, pipette offset, and pipette resistance are essential to the success of the recording. The calibration should be done for each micropipette that is used in this experiment.

- Connect the wires from the source and bulk regions of the silicon chip to variable power supply. Turn on power supply.

- Connect the wire from the drain to ground. The oscilloscope reads the voltage across resistor from drain to ground via banana and alligator clips.

- Ensure that both Channel 1 and Channel 2 traces appear on oscilloscope.

- Adjust the micromanipulator so that the tip of the sharp electrode penetrates the Retzius cell.

- Use Axoclamp software to inject current pulses into cell and record resulting signal. 


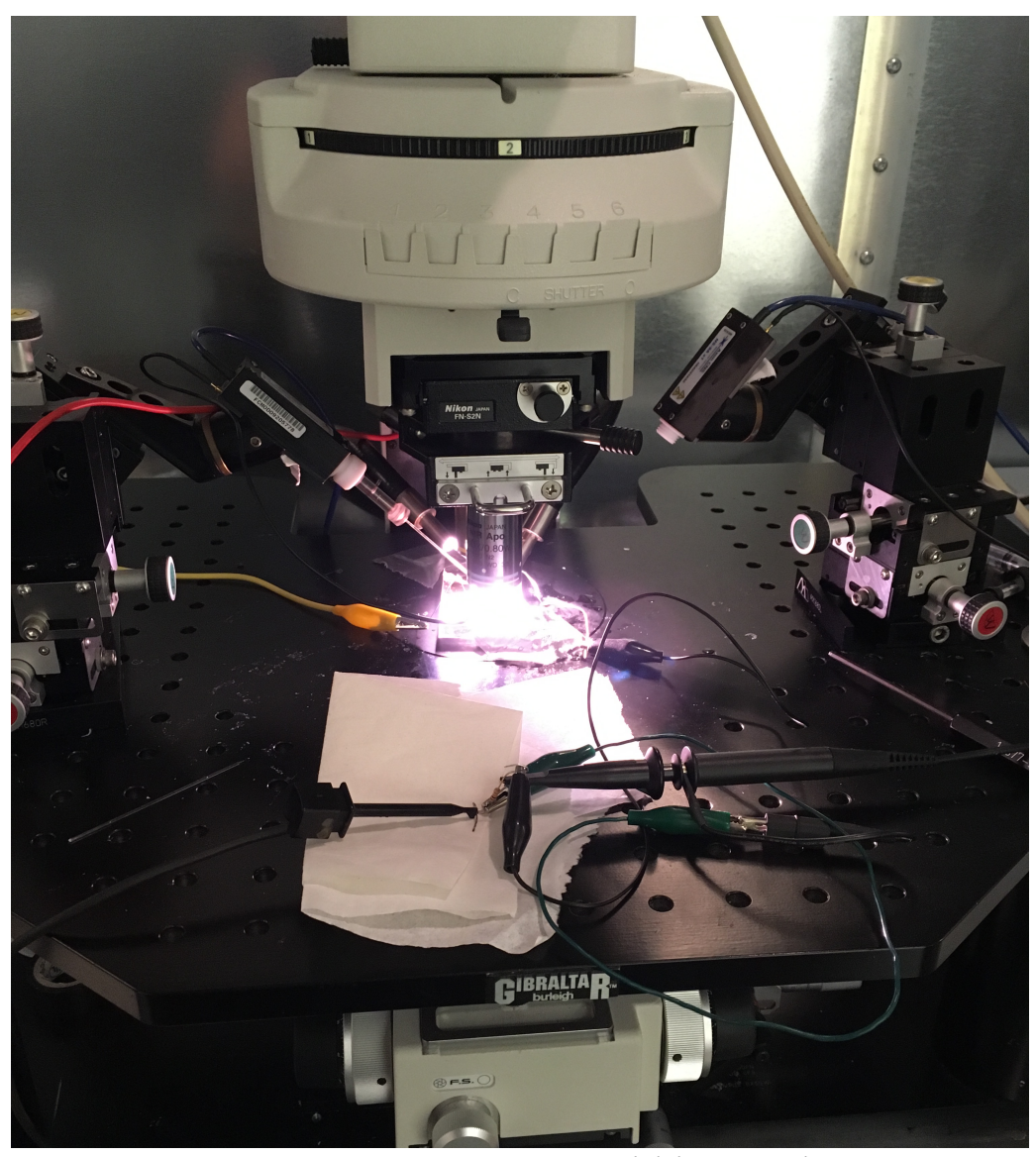

Figure 21. Setup within Faraday Cage 
CHAPTER 7. Results

\subsection{Oscilloscope Recording Results}

The first recording taken in each experiment trial used AxoClamp Commander $900 \mathrm{~A}$ to send a single pulse of $100 \mathrm{nA}$ of $10 \mathrm{~ms}$ duration. This stimulation served to prove that the microelectrode had sufficiently penetrated the cell and an adequate seal existed between the cell membrane and the transistor gate. Figure 22 shows a typical representation of this stimulation. The transistor voltage spikes approximately $2.50 \mathrm{~ms}$ before the action potential, a phenomenon that Fromherz observed in his 1998 experiments [4] and that is pictured in Figure 4.

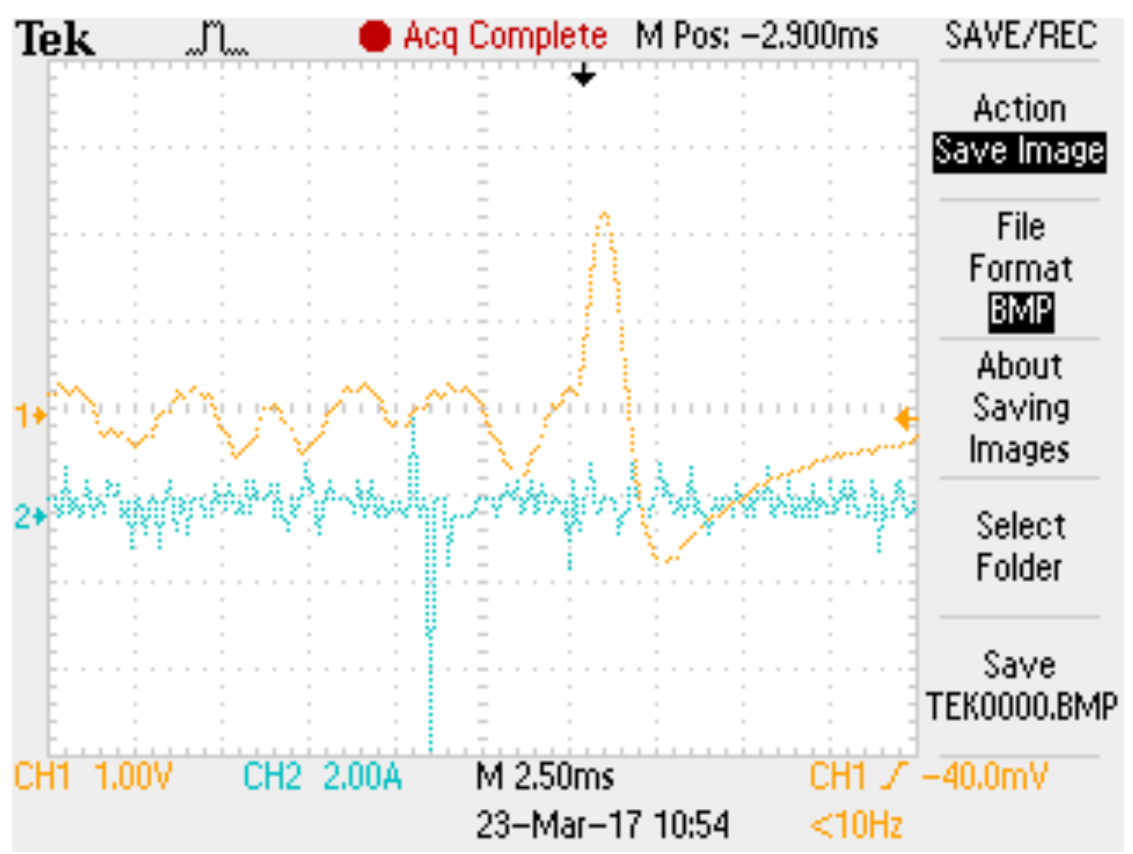

Figure 22. Electrical reading after stimulus. The yellow trace and blue trace represent the transmembrane and transistor voltage, respectively

The next stimulation implemented was a pulse train of two $50 \mathrm{~ms}$ pulses in the

Clampex 10.3 software. The amplitudes of the stimulus currents applied were .3nA, $.75 \mathrm{nA}, 1.5 \mathrm{nA}$ and $3 \mathrm{nA}$ to capture a range of responses. Though a change in 
transmembrane potential was observed, the form of this change resembled the rectangular stimulus pulses and not that of typical action potentials. These results are pictured in Figure 23. It should be noted that the series electrode resistance was not adequately calibrated out for this micropipette, which is why square waves appear in the cell responses in Figures 23 and 24. 

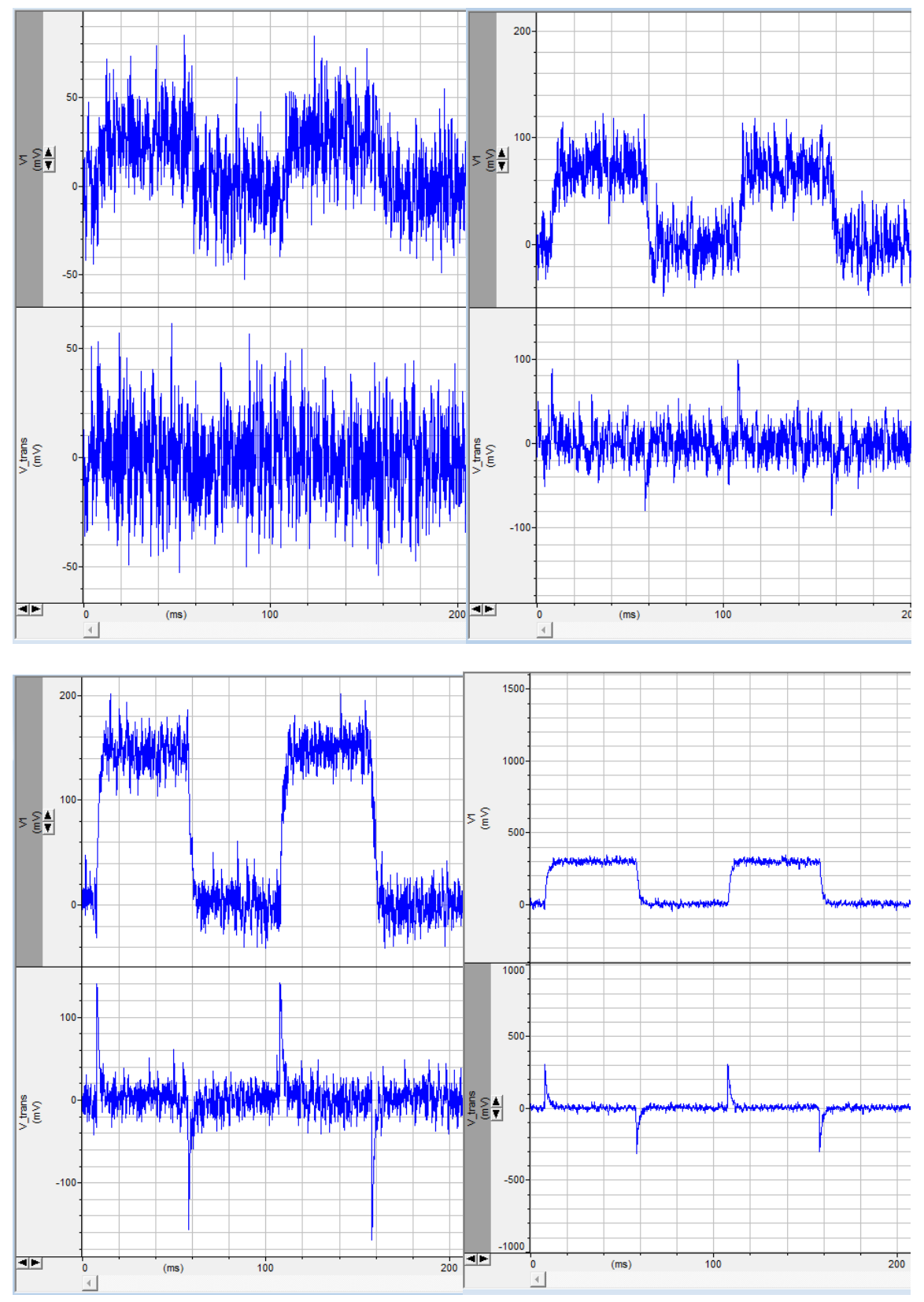

Figure 23. Oscilloscope traces resulting from $0.3, .75,1.5$, and $3 \mathrm{nA}$ stimuli, respectively.

$\mathrm{V} 1$ represents the change in membrane potential in milliVolts that results from the current injected into the Retzius cell; $\mathrm{V}$ trans represents the change in transistor voltage resulting from the change in membrane potential

After initially trying a two-pulse stimulus, a pulse train consisting of six $3 \mathrm{nA}$ current pulses was implemented. This yielded the strongest evidence of transistor gate 
current modulation as a result of Retzius cell action potential. Retzius cell action potentials are small compared to the large change in membrane potential resulting from the current stimuli, so Figure 24 shows a zoomed-in portion of the membrane potential and transistor voltage traces in addition to the raw trace.

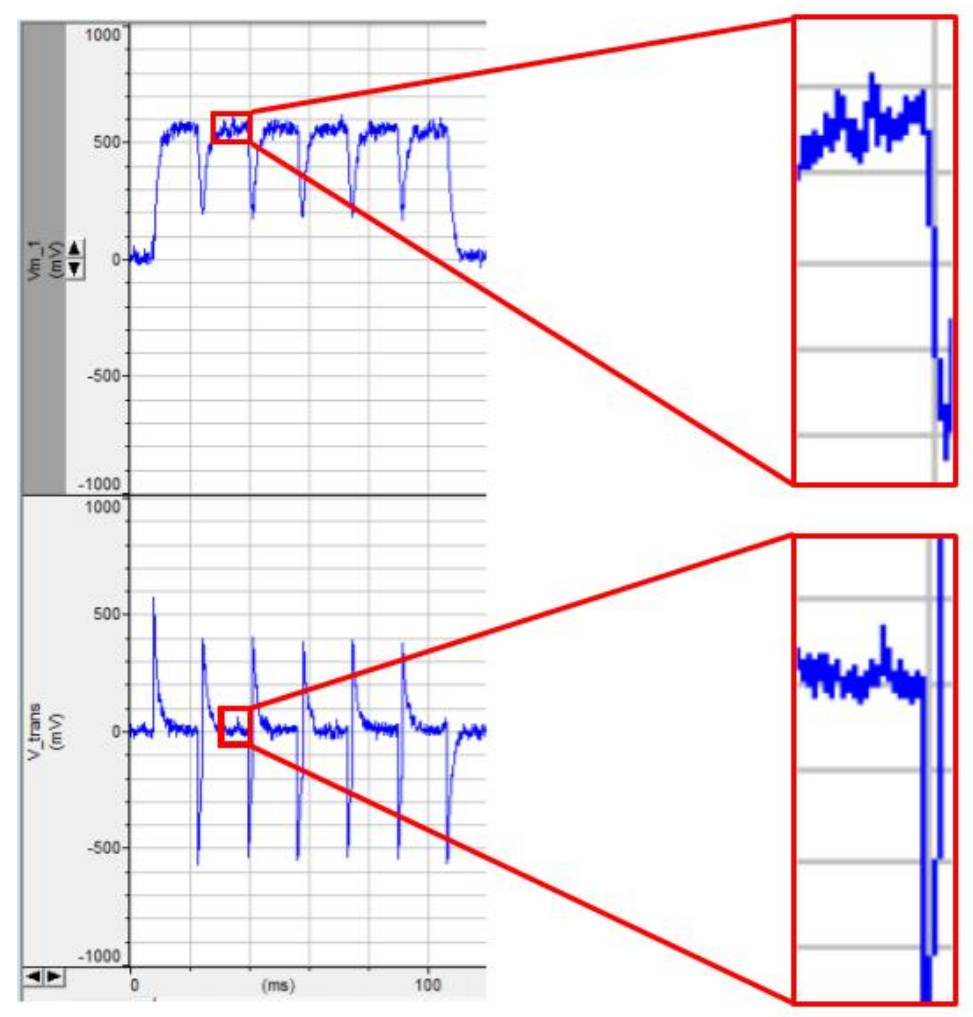

Figure 24. Electrical reading after 3nA stimulus; cell membrane potential on top and transistor voltage on bottom

Since the overarching goal of this thesis was replication, no statistical analysis of the data was required. 
CHAPTER 8. Discussion

The purpose of this thesis was to replicate Fromherz's 1991 experimental setup by studying the in vitro interface of a single leech Retzius cell with a MOSFET.

\subsection{Project Challenges}

\subsubsection{Physical geometry of device}

One of the most significant challenges this project presented was the physical geometry of the device. Fromherz's papers did not give specific or detailed information about the form of the device. The most concrete dimension that came out of reviewing the literature was that the diameter of the well above the transistor gate needed to be just large enough for the Retzius cell to fit without being too big and thereby negatively impacting the seal between the cell membrane and the transistor gate. The first few versions of the device achieved this, but failed when it came time to insert the microelectrode tip into the cell. The range of microelectrode positions was dictated by the limited space between the side of the device and the low position of the microscope. This led to designing the device to have a slimmer profile so the microelectrode could be successfully positioned for cell penetration.

\subsubsection{Visualization and cell maneuverability}

Most electrophysiology experiments are performed on whole ganglia, so there is limited literature specifically on the manipulation of single neurons. The size of the Retzius cell determined how the neuron treatment proceeded. The initial protocol was to pin the ganglion out using micropins in a small petri dish filled with silicone, perform the treatment with the collagenase/dispase solution, then transfer to the device and maneuver 
into the well. Neither a micropipettor nor the micro-dissecting forceps successfully accomplished this transfer, so the protocol was altered and the neuron treatment occurred on the device itself. Glass holding micropipettes were pulled with an inner diameter just slightly smaller than the Retzius cell diameter, which were the key to maneuvering the cell into place. Additionally from a purely qualitative perspective, some Retzius cells appeared to be more opaque than others, which hindered identification and manipulation.

\subsubsection{Neuron treatment}

The primary disadvantage of the neuron treatment occurring in the same solution and vicinity of the transistor gate well was that collagenase/dispase was present even after the ganglion had been dissected and into the experimentation stimulations. It is very possible that these enzymes continued to degrade the Retzius cell's membrane. Possible consequences of this may have been the failure to create action potentials with small amplitude pulses delivered by the Clampex software.

\subsection{Concluding Remarks}

The results indicate that change in transmembrane potential can be correlated with change in transistor gate voltage. Before the experimental phase of this project began, it was decided that successful replication of Fromherz's 1991 would be defined in this exercise as any level of transistor signal resulting from a Retzius cell action potential. So in that sense, this project can be considered successful. Of course, our experimental data and results have potential to be improved. The results presented in Chapter 7 contain noise that hinders the identification of action potentials and resultant transistor gate voltage spikes. Optimum calibration of the electrophysiology equipment was not 
achieved due to experimenter error and contributed to the sub-optimal quality of the data. Another iteration of this project in the future could include exploring the junctions presented in Fromherz's 1998 work, in which the physical orientation of the Retzius cell had temporal effects on the response of the transistor voltage. 


\section{References}

1. Fromherz, P et al. "A Neuron-Silicon Junction: A Retzius Cell of the Leech on an Insulated-Gate Field-Effect Transistor.” Science 252.5010 (1991): 1290. Web.

2. Fromherz, Peter, Carsten O. Müller, and Rolf Weis. "Neuron Transistor: Electrical Transfer Function Measured by the Patch-Clamp Technique.” Physical Review Letters 71.24 (1993): 4079-4082. Print.

3. Weis, Rolf, Bernt Müller, and Peter Fromherz. "Neuron Adhesion on a Silicon Chip Probed by an Array of Field-Effect Transistors." Physical Review Letters 76.2 (1996): 327-330. Print.

4. Schätzthauer, Richard, and Peter Fromherz. "Neuron-silicon Junction with VoltageGated Ionic Currents.” European Journal of Neuroscience 10.6 (1998): 19561962. Web.

5. "Electrophysiology." Wikipedia. Wikimedia Foundation, 12 Apr. 2017. Web. 29 Apr. 2017.

6. Hewitt, John. "How many types of neurons do we need to define?" Medical Xpress medical research advances and health news. Medical Xpress, 20 Aug. 2013. Web. 29 Apr. 2017.

7. "MOSFET." Wikipedia. Wikimedia Foundation, 25 Apr. 2017. Web. 29 Apr. 2017.

8. "Cell membrane." Encyclopcedia Britannica. Encyclopædia Britannica, inc., n.d. Web. 29 Apr. 2017.

9. "Introduction to Computational Neuroscience." Intro to CNS part I. University of Colorado, Boulder, 17 June 2008. Web. 29 Apr. 2017.

10. OpenStax. "Anatomy and Physiology." Introduction | Anatomy and Physiology. OpenStax, 06 Mar. 2013. Web. 29 Apr. 2017.

11. Ellis, Lisa, Joe Frankel, and Ryan Krauss. "An Overview of Transistors." N.p., n.d. Web. 29 Apr. 2017.

$<$ http://www.imdl.gatech.edu/ryan/course_work_portfolio/Mechatronics/Transisto rs_Student_Lecture_Fal12002.pdf $>$. 
12. Brodfuehrer, Peter D., and Maria Stella E. Thorogood. "Identified Neurons and Leech Swimming Behavior." Progress in Neurobiology 63.4 (2001): 371-381. Web.

13. "Leech Neuroanatomy." Leech Swimming. N.p., n.d. Web. 29 Apr. 2017.

14. Higgins, Alexandra et al. "Differential Modulation of Nociceptive versus NonNociceptive Synapses by Endocannabinoids.” Molecular Pain 9 (2013): 26-26. Web.

15. Titlow, Josh et al. "Intracellular Recording, Sensory Field Mapping, and Culturing Identified Neurons in the Leech, Hirudo Medicinalis." Journal of Visualized Experiments : JoVE 81 (2013): 50631. PMC. Web. 1 May 2017.

16. User, Super. "Leech Mobile Home." Leeches U.S.A. Leeches U.S.A., n.d. Web. 01 May 2017.

17. Dietzel, I D, P Drapeau, and J G Nicholls. "Voltage Dependence of 5Hydroxytryptamine Release at a Synapse between Identified Leech Neurones in Culture.” The Journal of Physiology 372 (1986): 191-205. Print.

18. Sutter Instrument. "Pipette Cookbook 2015: P-97 \& P-1000 Micropipette Pullers." Vers. E. 2015. www.sutter.com. 29 April 2017 $<$ https://www.sutter.com/PDFs/pipette_cookbook.pdf $>$.

19. G. G. Matthews, Cellular physiology of nerve and muscle, 4th ed. Osney Mead, Oxford: Blackwell Pub., 2003. 


\section{Appendices}

Appendix A: Pulled Glass Micropipette Recipes

Both recipes use a $2.5 \mathrm{~mm} \times 2.5 \mathrm{~mm}$ Box Filament with $1.5 \mathrm{~mm} \times 0.86 \mathrm{~mm}$ Thick Walled Glass with Filament micropipettes.

\section{Table 2. Holding Pipette Recipe}

\begin{tabular}{|l|l|l|l|l|}
\hline Heat & Pull & Velocity & Delay & Pressure \\
\hline 510 & 0 & 150 & 0 & 200 \\
\hline
\end{tabular}

In order to complete holding pipette fabrication, it is necessary to break the tip approximately halfway up the taper. This can be accomplished by scoring with another piece of glass and pushing through the scored region with a gentle, continuous motion, as shown in Figure 25.
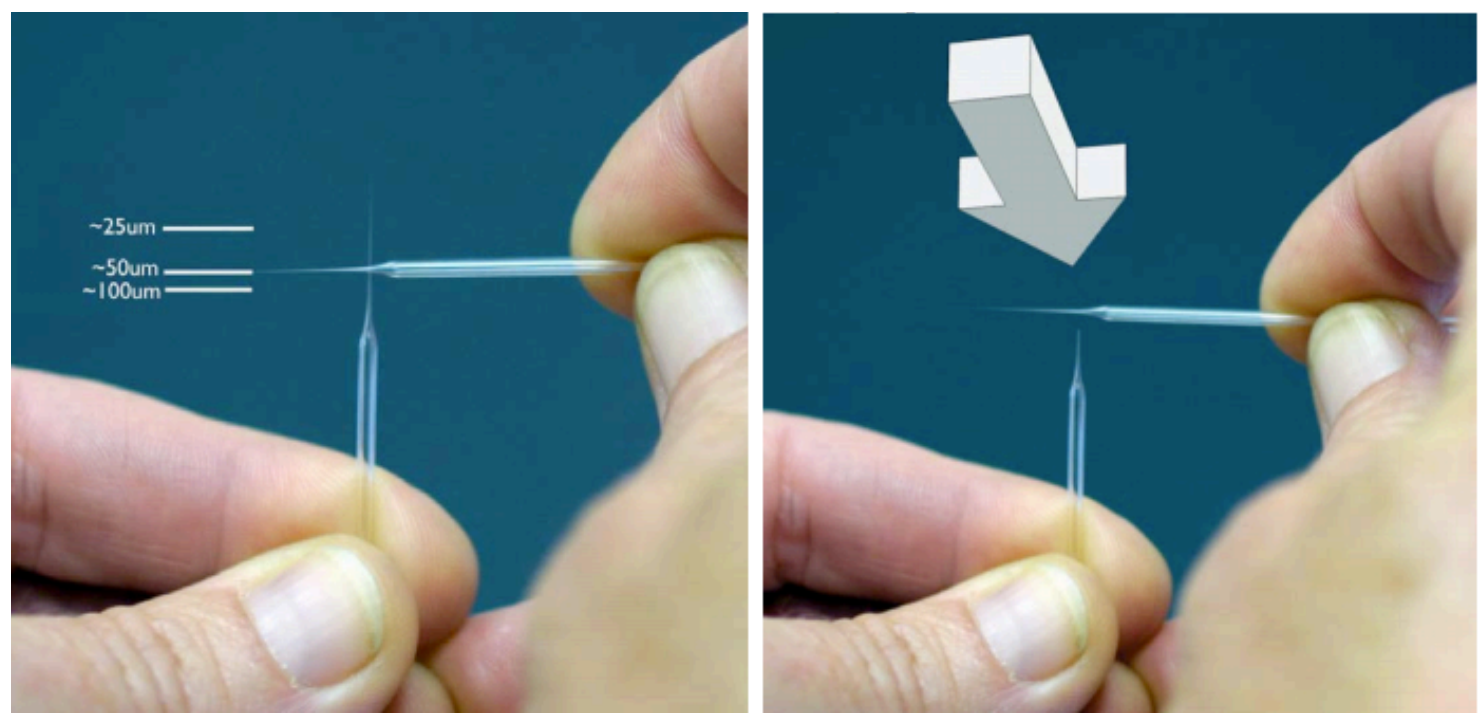

Figure 25. Correct technique for breaking the holding pipette tip [18]

Table 3. Intracellular Microelectrode Pipette Recipe

\begin{tabular}{|l|l|l|l|l|}
\hline Heat & Pull & Velocity & Delay & Pressure \\
\hline 480 & 70 & 80 & 100 & 500 \\
\hline
\end{tabular}


Appendix B. Solution Preparations

Neuron Culture Solution

- $100 \mathrm{mg}$ of collagenase/dispase

- $4.5 \mathrm{~mL}$ of L-15 solution

- $1 \mathrm{~mL}$ of gentamicin

- $2 \mathrm{~mL}$ of fetal calf serum (FCS)

Isotonic Leech Bath

- 1 gallon distilled water

- 2 grams HIRUDO salt from Leeches USA

Leech Ringer's Solution

- 1 Liter of distilled water mixed with:

- $38 \mathrm{~mL}$ of 3 molar $\mathrm{NaCl}$

- $1.8 \mathrm{~mL}$ of 1 molar $\mathrm{CaCl}_{2}$

- $4 \mathrm{~mL}$ of 1 molar $\mathrm{KCl}$

○ $50 \mathrm{~mL}$ of 0.2 molar Tris maleate 


\section{Appendix C. Leech Carcass Disposal}

1. Using scalpel, sever the rostral and caudal regions from the rest of the body.

2. Unpin the leech from the silicone-filled petri dish.

3. Deposit leech pieces into red biohazard bag located in lab freezer.

4. Rinse petri dish with water and let dry. 


\section{Appendix D. Matlab code modeling gating variable and transmembrane potential response to a step current excitation}

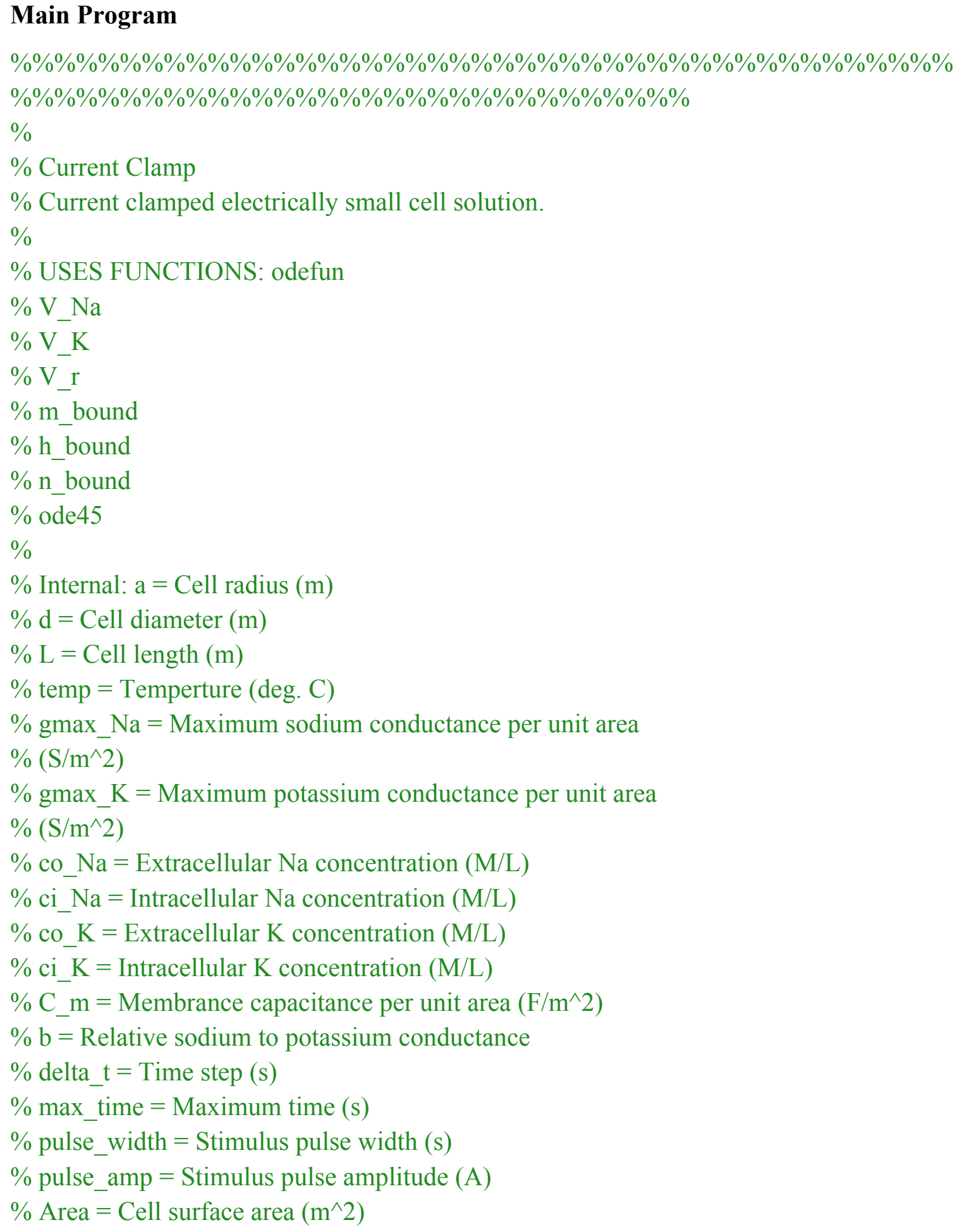




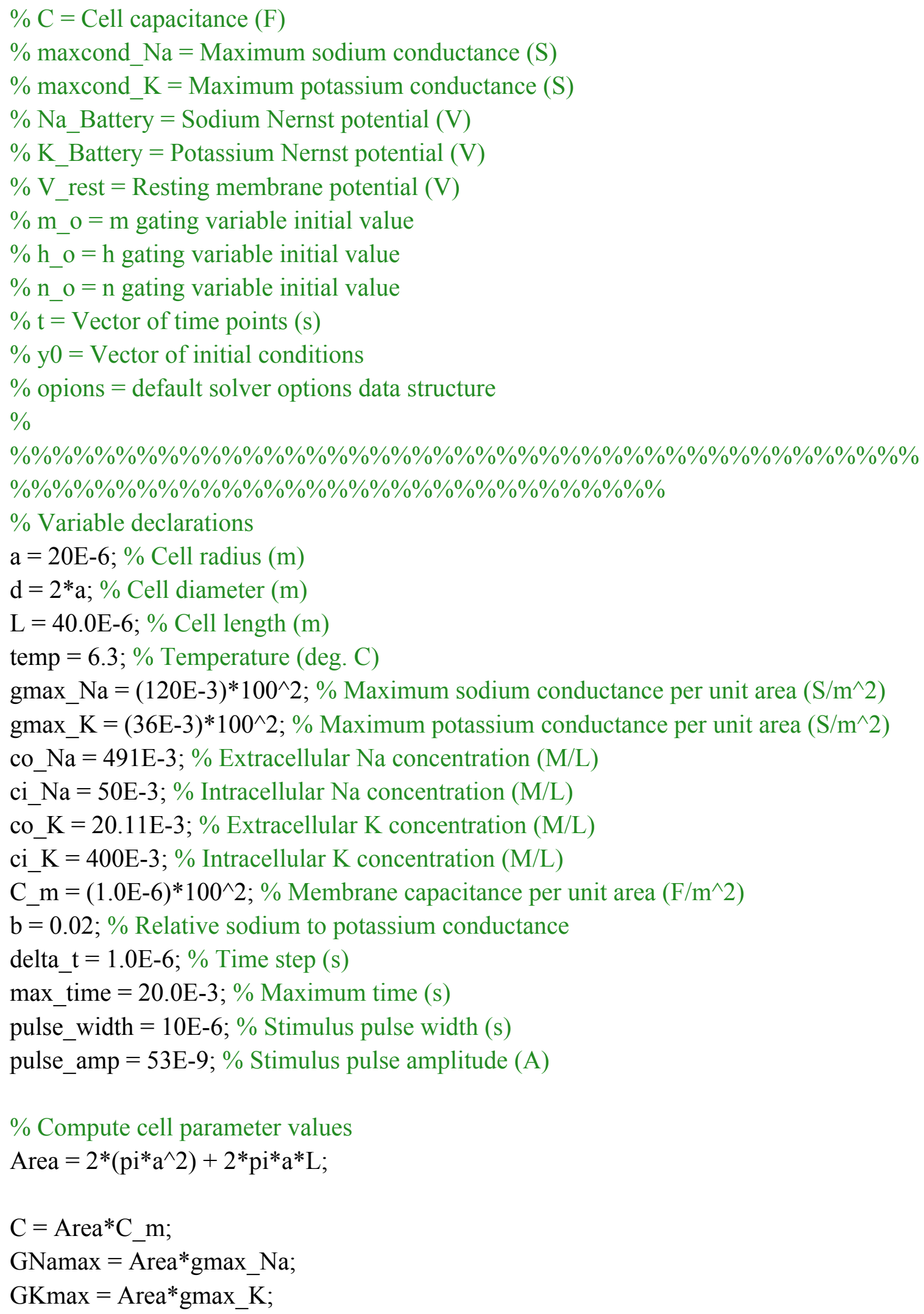




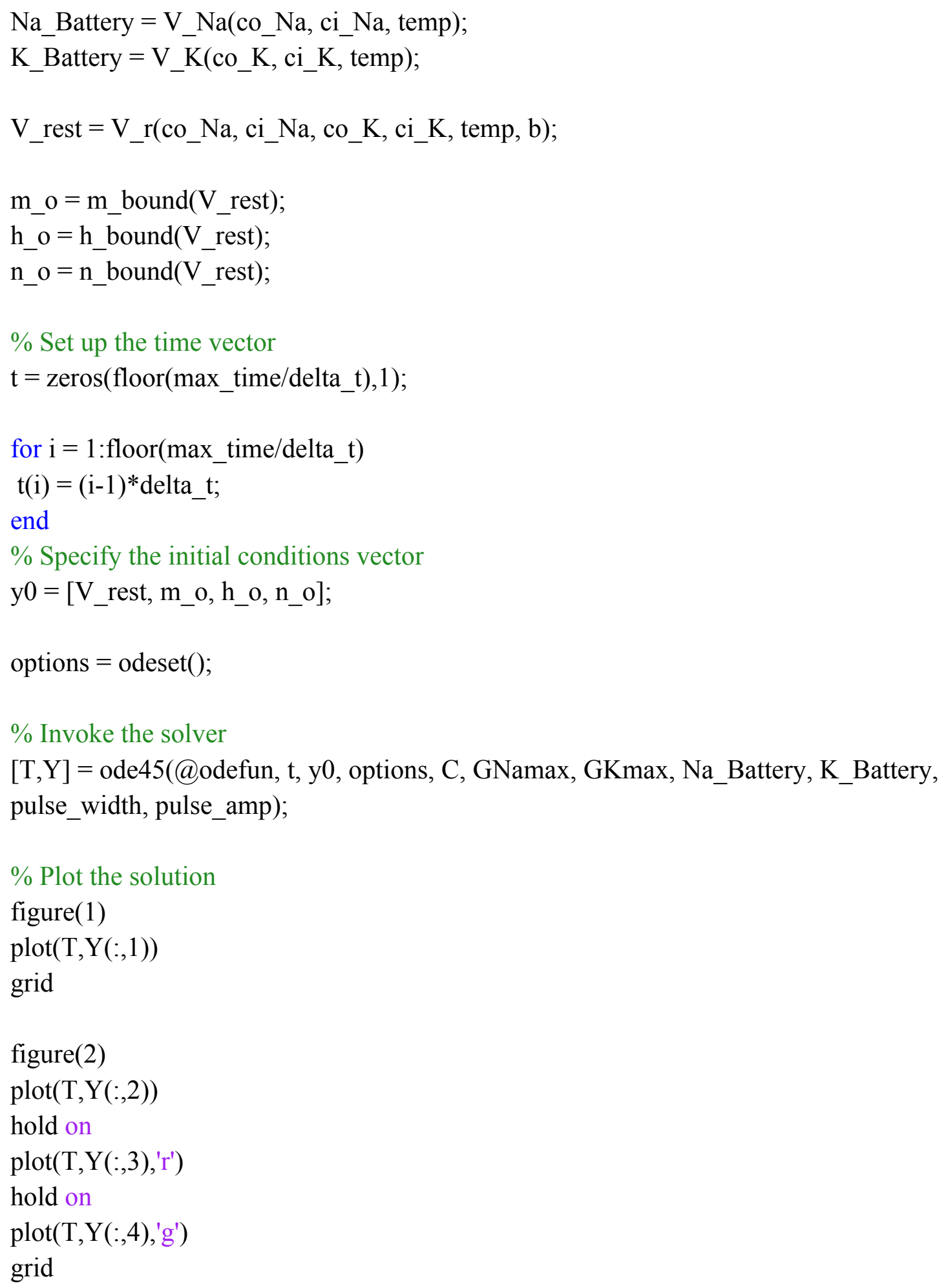




\section{User-Defined Functions used in Main Program}

$\%$ Function: alpha_h

$\%$

$\%$ Computes the value of the alpha rate constant for the $\mathrm{h}$ gating

$\%$ variable.

$\%$

$\%$ Inputs: $\mathrm{Vm}=$ Membrane Potential (V)

$\%$

$\%$ Returns: $a h=$ Returns the alpha rate constant for the $h$

$\%$ gating variable $(1 / \mathrm{ms})$

$\%$

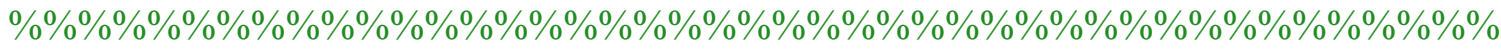
\% \% \% \% \% \% \% \% \% \% \% \% \% \% \% \% \% \% \% \% \% \% \% \% \% \% \% \% \% \% \%

function ah $=$ alpha_h(Vm)

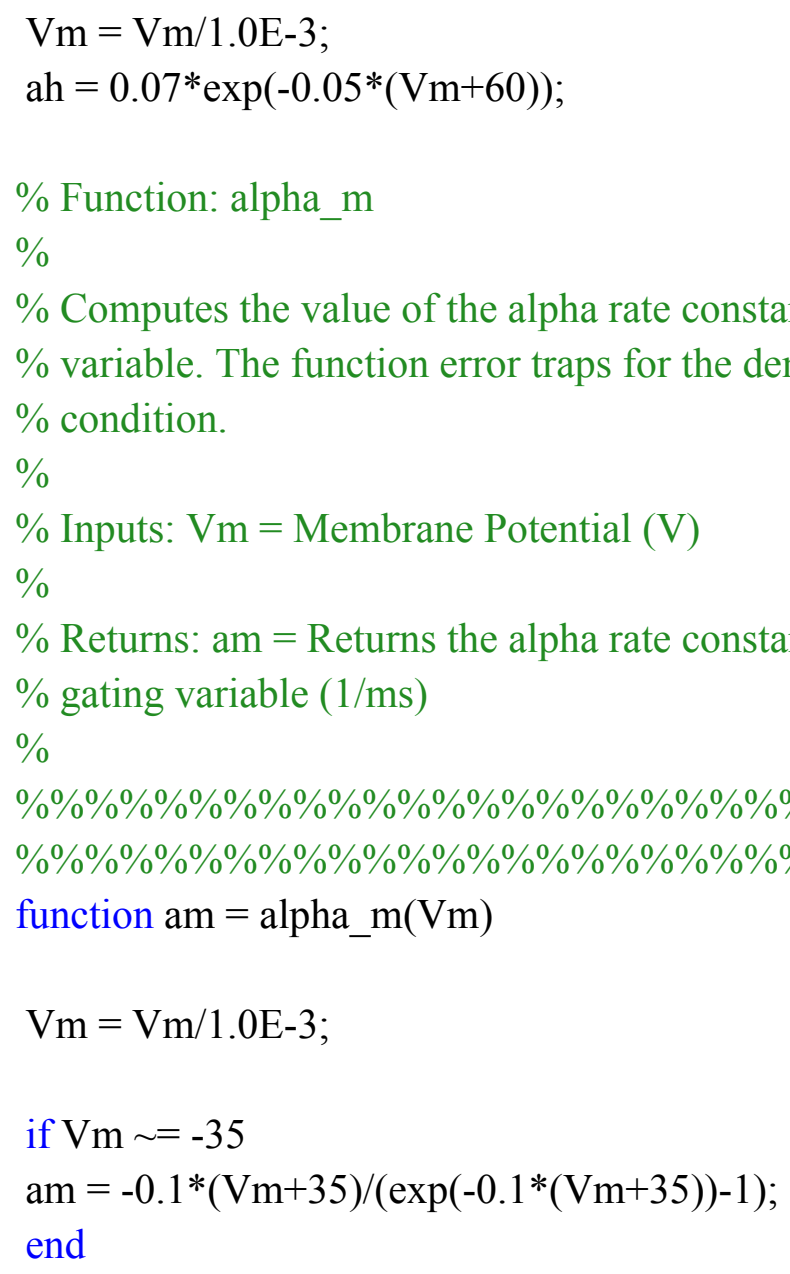




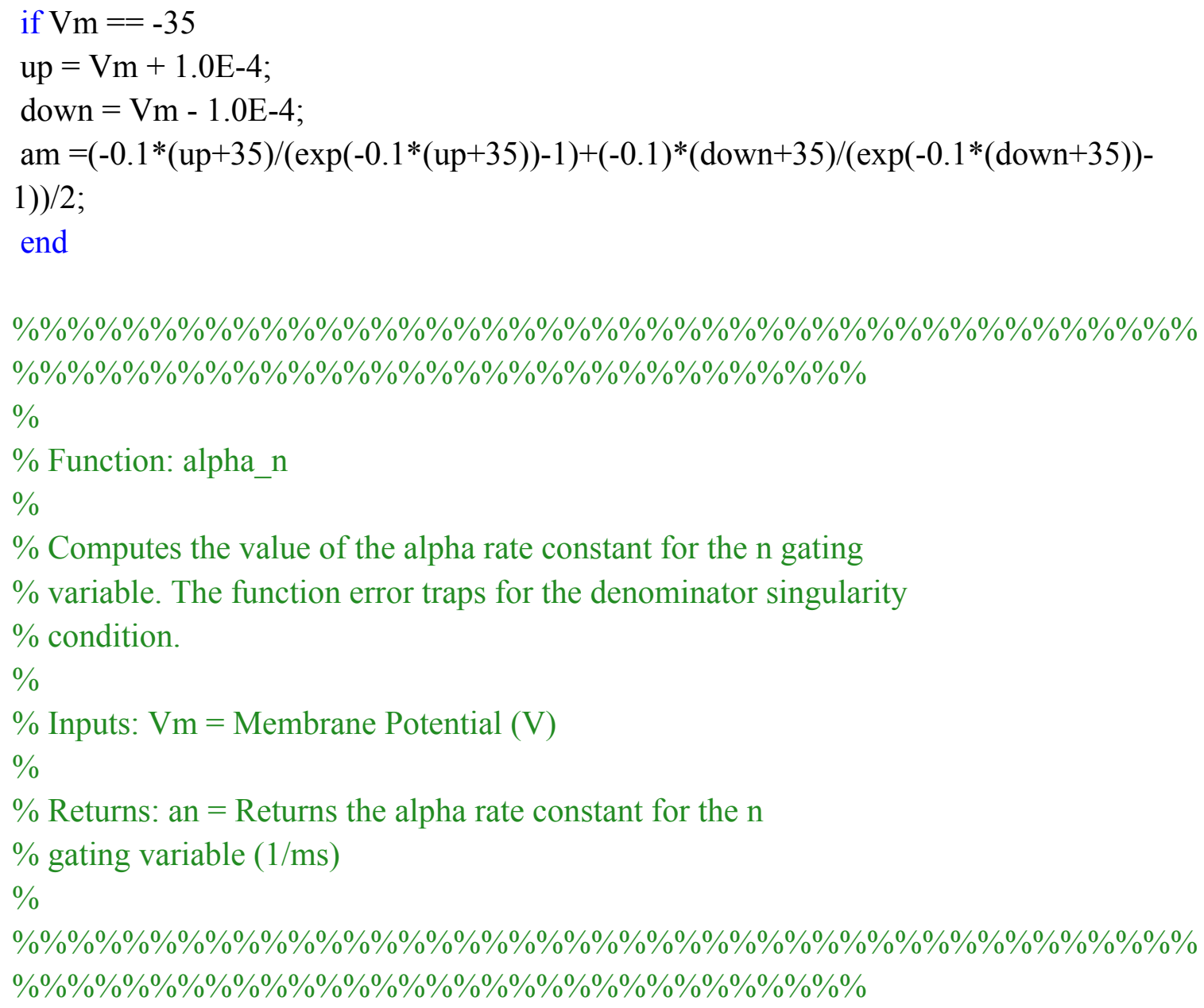




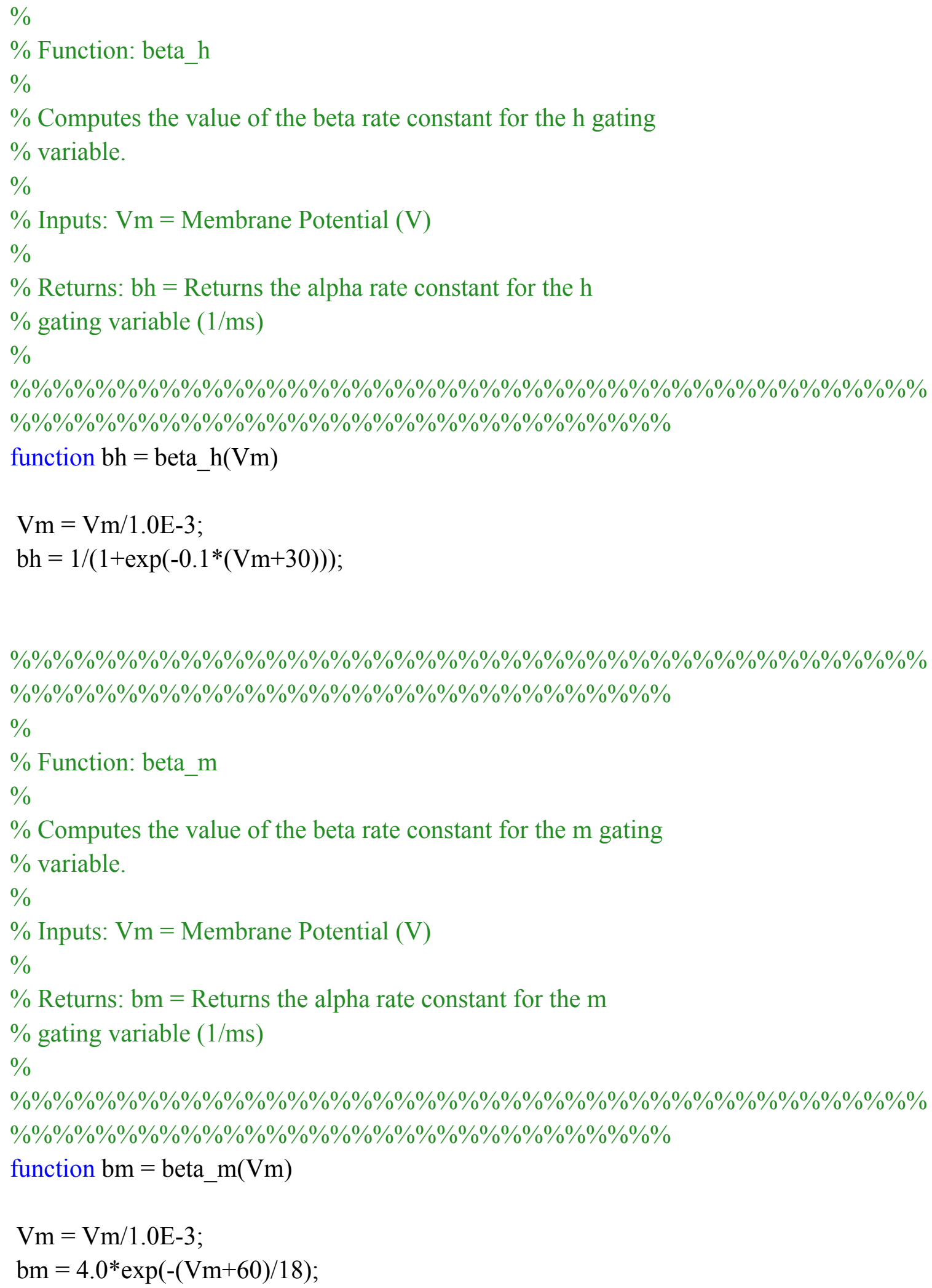




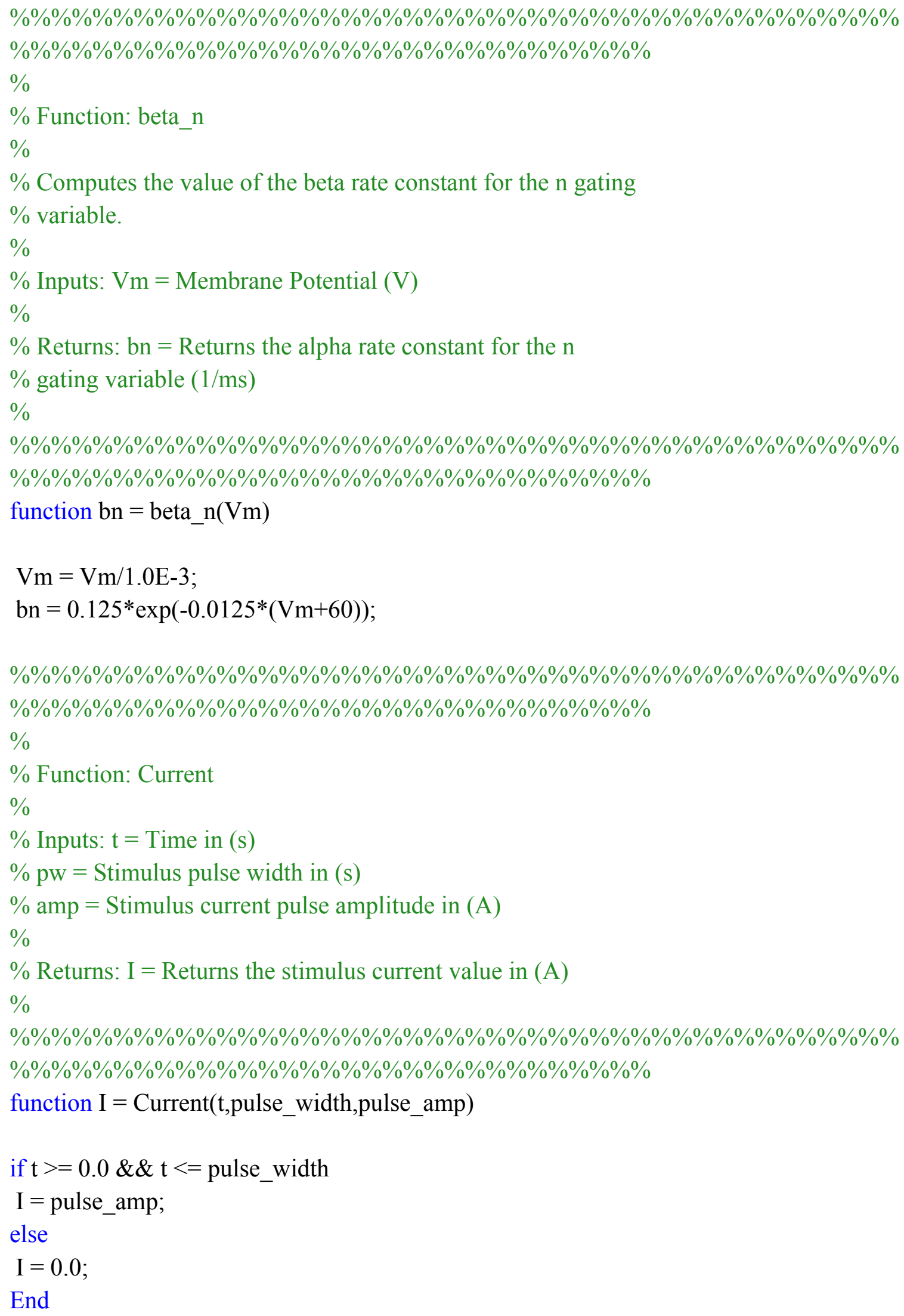




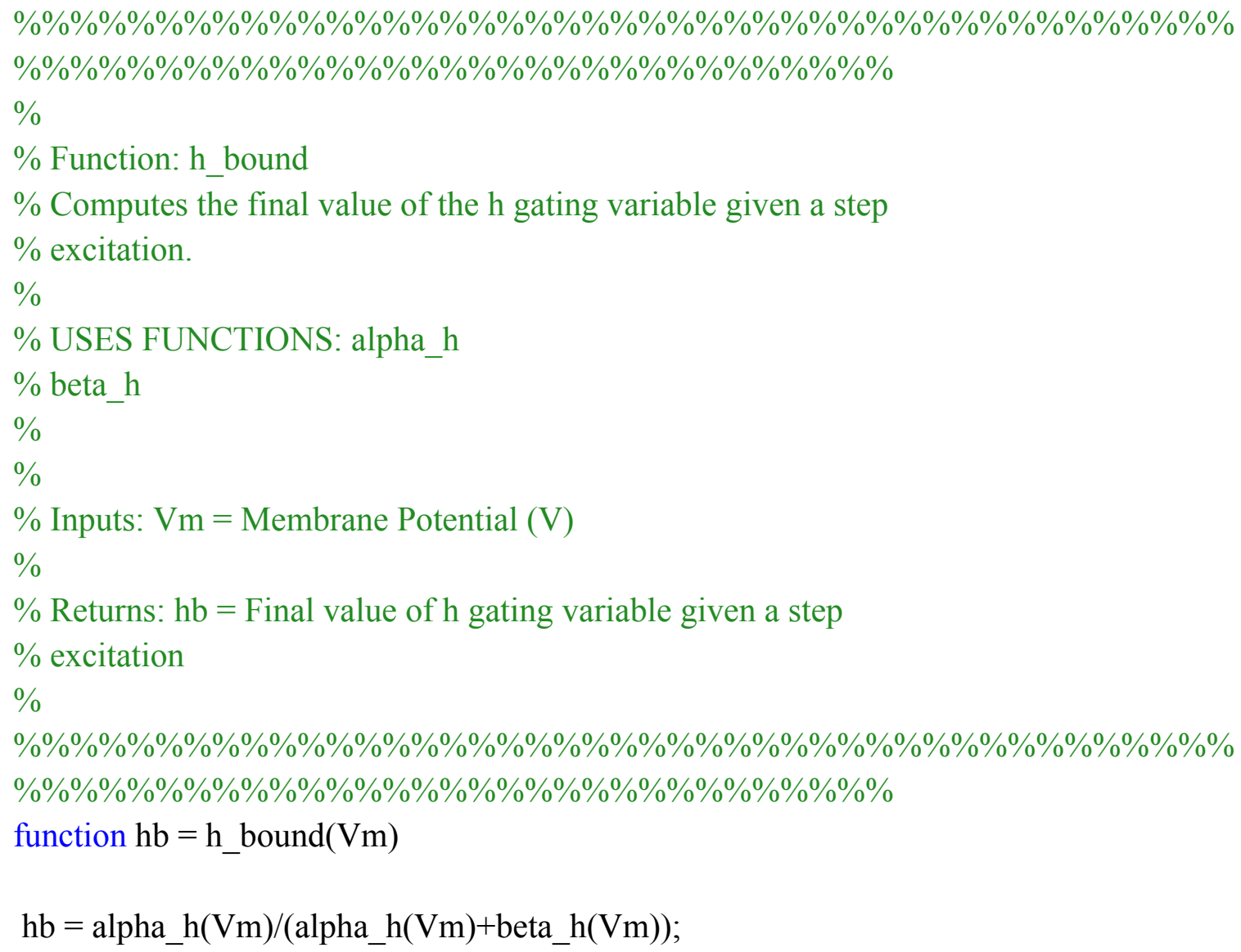




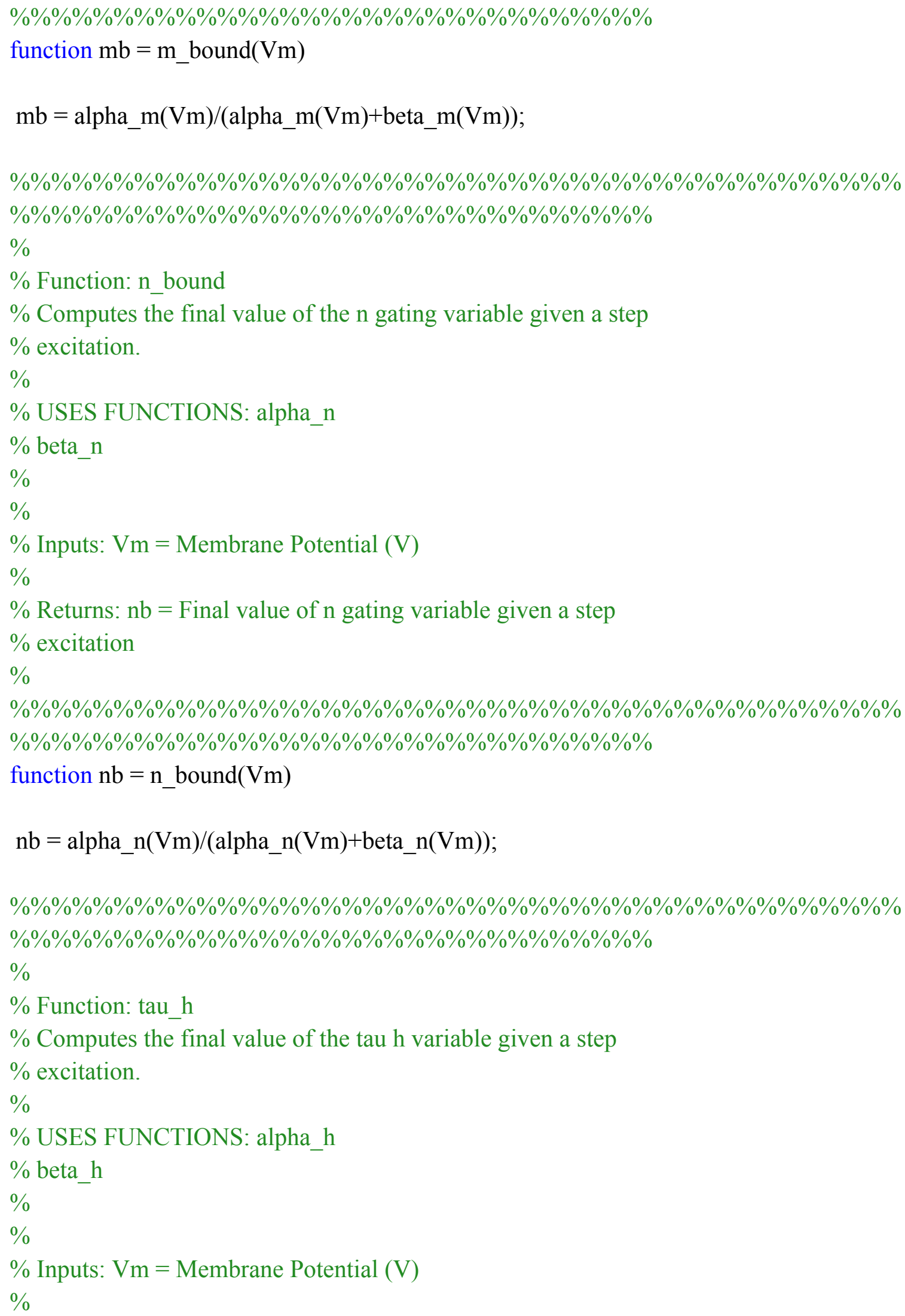


$\%$ Returns: tauh $=$ Final value of tauh gating variable given a step

$\%$ excitation

$\%$

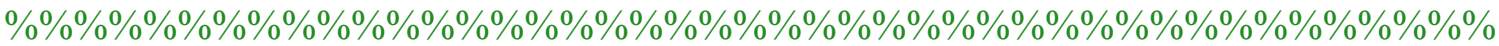

\% $\% \% \% \% \% \% \% \% \% \% \% \% \% \% \% \% \% \% \% \% \% \% \% \% \% \% \% \% \% \%$

function tauh $=$ tau_h(Vm)

$\operatorname{tauh}=1 /($ alpha_h $(\mathrm{Vm})+$ beta_h(Vm) $)$;

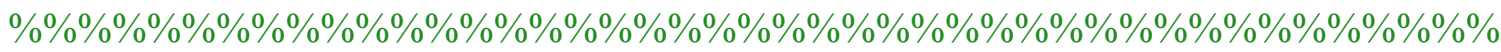
\% $\% \% \% \% \% \% \% \% \% \% \% \% \% \% \% \% \% \% \% \% \% \% \% \% \% \% \% \% \% \% \% \%$

$\%$

$\%$ Function: tau m

$\%$ Computes the final value of the tau $\mathrm{m}$ variable given a step

$\%$ excitation.

$\%$

$\%$ USES FUNCTIONS: alpha_m

$\%$ beta $m$

$\%$

$\%$

$\%$ Inputs: $\mathrm{Vm}=$ Membrane Potential (V)

$\%$

$\%$ Returns: taum $=$ Final value of taum gating variable given a step

$\%$ excitation

$\%$

\%\%\%\%\%\%\%\%\%\%\%\%\%\%\%\%\%\%\%\%\%\%\%\%\%\%\%\%\%\%\%\%\%\%\%\%\%\%\%\%\%\%\%\%\%

\% $\% \% \% \% \% \% \% \% \% \% \% \% \% \% \% \% \% \% \% \% \% \% \% \% \% \% \% \% \% \%$

function taum $=$ tau_m(Vm)

taum $=1 /($ alpha_m $(\mathrm{Vm})+$ beta_m $(\mathrm{Vm}))$

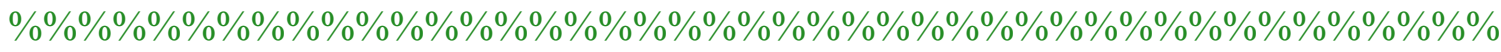

\%\%\%\%\%\%\%\%\%\%\%\%\%\%\%\%\%\%\%\%\%\%\%\%\%\%\%\%\%\%\%

$\%$

$\%$ Function: tau $\mathrm{n}$

$\%$ Computes the final value of the tau $\mathrm{n}$ variable given a step

$\%$ excitation.

$\%$

$\%$ USES FUNCTIONS: alpha_n

$\%$ beta_n 


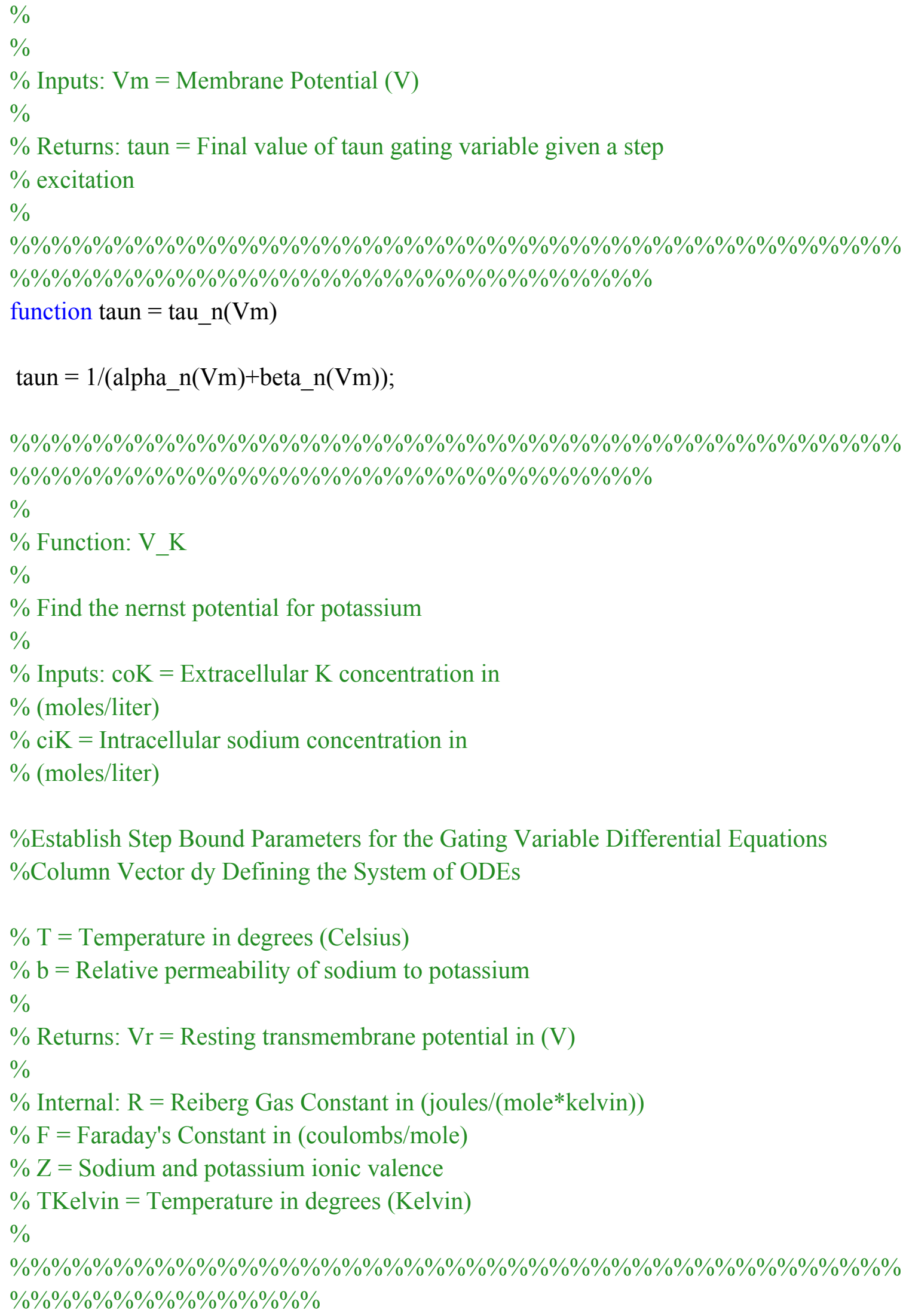




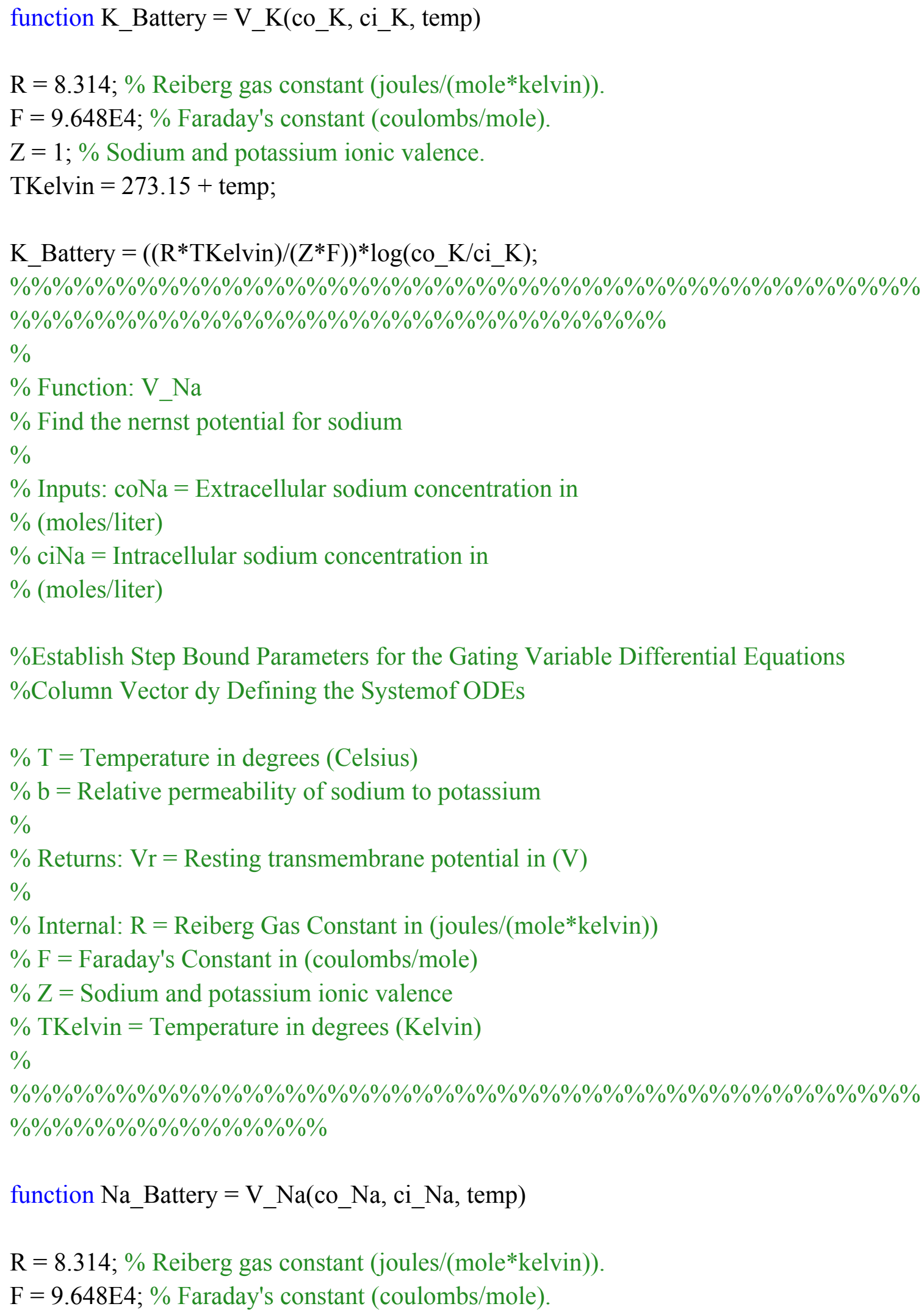


$\mathrm{Z}=1 ; \%$ Sodium and potassium ionic valence.

TKelvin $=273.15+$ temp;

\section{Na_Battery $=\left(\left(\mathrm{R}^{*} \mathrm{TKelvin}\right) /(\mathrm{Z} * \mathrm{~F})\right) * \log \left(\mathrm{co} \_\mathrm{Na} / \mathrm{ci} \_\mathrm{Na}\right)$;}

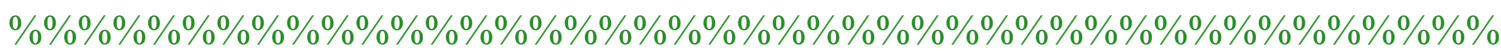
\%\%\%\%\%\%\%\%\%\%\%\%\%\%\%\%\%\%\%\%\%\%\%\%\%\%\%\%\%\%\%

$\%$

$\%$ Function: $\mathrm{V}$ r

$\%$

$\%$ Find the resting membrane potential using

$\%$ the Goldmann Equation.

$\%$

$\%$ Inputs: coNa $=$ Extracellular sodium concentration in

$\%$ (moles/liter)

$\% \mathrm{ciNa}=$ Intracellular sodium concentration in

$\%$ (moles/liter)

$\% \operatorname{coK}=$ Extracellular potassium concentration in

$\%$ (moles/liter)

$\% \operatorname{ciK}=$ Intracellular potassium concentration in

$\%$ moles/liter

$\%$ Establish Step Bound Parameters for the Gating Variable Differential Equations

$\%$ Column Vector dy Defining the Systemof ODEs

$\% \mathrm{~T}=$ Temperature in degrees (Celsius)

$\% \mathrm{~b}=$ Relative permeability of sodium to potassium

$\%$

$\%$ Returns: $\mathrm{Vr}=$ Resting transmembrane potential in $(\mathrm{V})$

$\%$

\% Internal: $\mathrm{R}=$ Reiberg Gas Constant in (joules/(mole*kelvin))

$\% \mathrm{~F}=$ Faraday's Constant in (coulombs/mole)

$\% \mathrm{Z}=$ Sodium and potassium ionic valence

$\%$ TKelvin $=$ Temperature in degrees (Kelvin)

$\%$

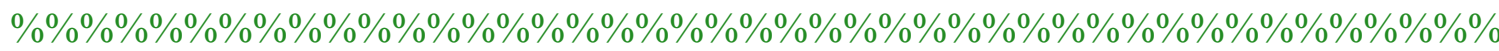

\% \% \% \% \% \% \% \% \% \% \% \% \% \% \% \%

function $\mathrm{V} \_$rest $=\mathrm{V} \_$r(coNa,ciNa,coK, ciK, T,b)

$\mathrm{R}=8.314 ; \%$ Reiberg gas constant (joules/(mole*kelvin)). 
$\mathrm{F}=9.648 \mathrm{E} 4 ; \%$ Faraday's constant (coulombs/mole).

$\mathrm{Z}=1 ; \%$ Sodium and potassium ionic valence.

TKelvin $=273.15+\mathrm{T}$

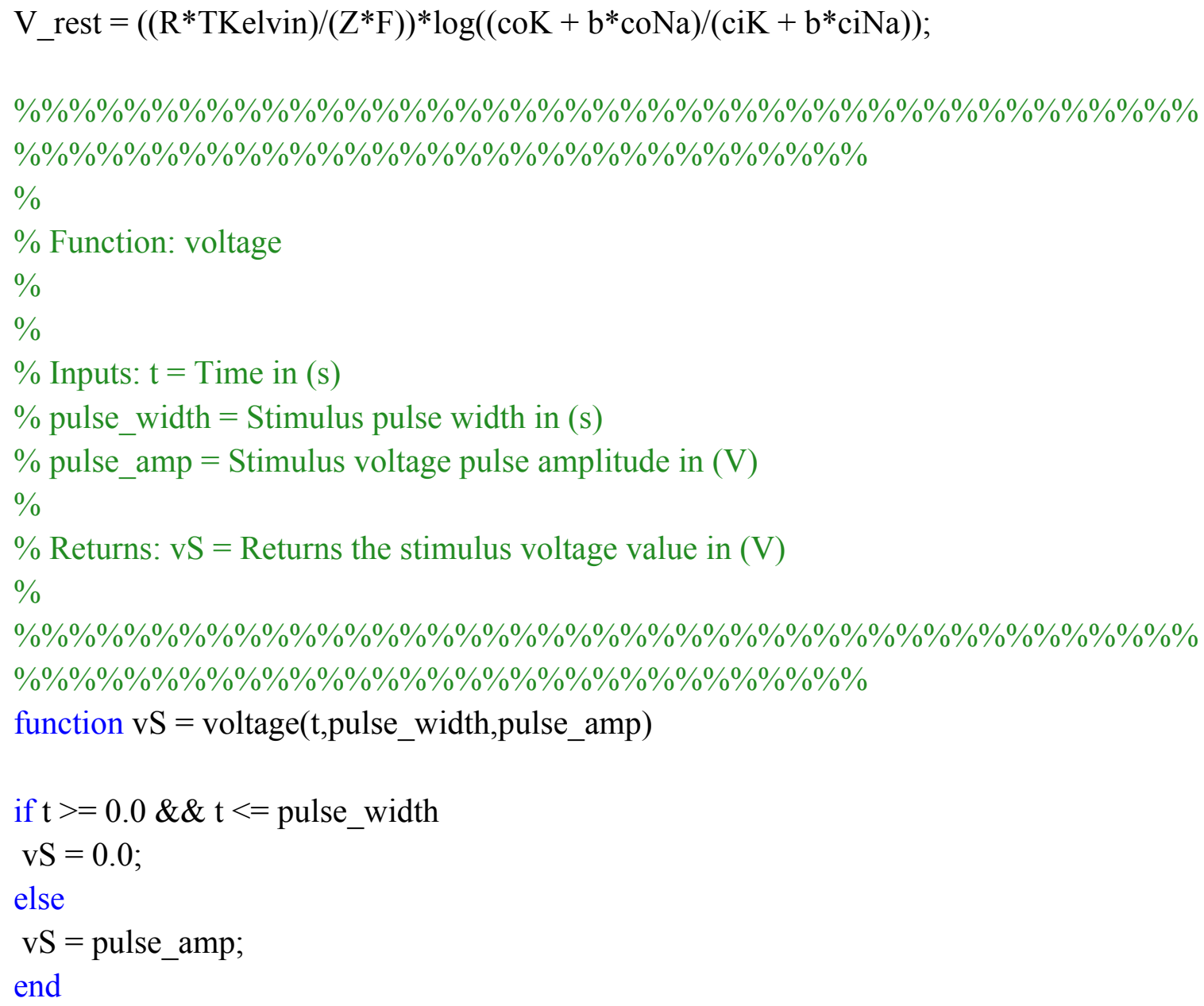




\section{ODE solver for Part 1:}

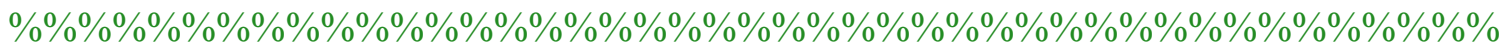

\%\%\%\%\%\%\%\%\%\%\%\%\%\%\%\%\%\%\%\%\%\%\%\%\%\%\%\%\%\%\%

$\%$ Odefunction

$\%$

$\%$ Implement the system of nonlinear ordinary differential equations

$\%$ associated with the problem presented in Section 6.1.

$\%$

$\%$ USES FUNCTIONS: $m$ bound

$\%$ h bound

$\% \mathrm{n}$ bound

$\%$ tau_m

$\%$ tau_h

$\%$ tau n

$\%$ Current

$\%$

$\%$ Inputs: $\mathrm{t}=$ current time point being evaluated in (s)

$\% \mathrm{y}=$ previous value of the ODE variables, VECTOR

$\% \mathrm{Cm}=$ membrane capacitance in $(\mathrm{F})$

$\%$ GNamax $=$ maximum sodium conductance in $(\mathrm{S})$

$\% \mathrm{GKmax}=$ maximum potassium conductance in $(\mathrm{S})$

$\% \mathrm{VNa}=$ sodium Nernst potential in $(\mathrm{V})$

$\% \mathrm{VK}=$ potassium Nernst potential in $(\mathrm{V})$

$\%$ pulse_width $=$ stimulus pulse width in (s)

$\%$ pulse_amp $=$ stimulus pulse amplitude in (A)

$\%$

$\%$ Returns: $d y=$ first derivative value of ODE variables

$\%$

$\%$ Internal: $\mathrm{mf}=$ step bounded value of $\mathrm{m}$ gating variable

$\% \mathrm{hf}=$ step bounded value of $\mathrm{h}$ gating variable

$\% \mathrm{nf}=$ step founded value of $\mathrm{n}$ gating variable

$\%$ taum $=$ step rise time value of $\mathrm{m}$ gating variable

$\%$ tauh $=$ step rise time value of $\mathrm{h}$ gating variable

$\%$ taun $=$ step rise time value of $n$ gating variable

$\%$

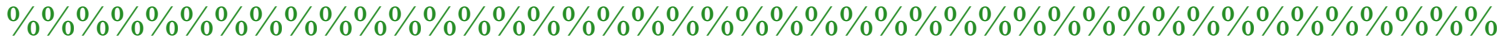
\%\%\%\%\%\%\%\%\%\%\%\%\%\%\%\%\%\%\%\%\%\%\%\%\%\%\%\%\%\%\%\%\%

function dy $=$ odefun $\left(\mathrm{t}, \mathrm{y}, \mathrm{Cm}, \mathrm{GNamax}, \mathrm{GKmax}, \mathrm{Na} \_\right.$Battery, K_Battery, pulse_width, pulse_amp) 


$$
\begin{aligned}
& \mathrm{mf}=\mathrm{m} \_ \text {bound }(\mathrm{y}(1)) ; \\
& \mathrm{hf}=\mathrm{h} \_ \text {bound }(\mathrm{y}(1)) ; \\
& \mathrm{nf}=\mathrm{n} \text { _bound }(\mathrm{y}(1)) ; \\
& \text { taum = tau_m(y(1)); } \\
& \text { tauh = tau_h }(\mathrm{y}(1)) ; \\
& \text { taun = tau_n }(\mathrm{y}(1)) ;
\end{aligned}
$$

$\mathrm{dy}=\left[-(\mathrm{GKmax} / \mathrm{Cm}) *\left(\mathrm{y}(4)^{\wedge} 4\right) *\left(\mathrm{y}(1)-\mathrm{K} \_\right.\right.$Battery $)-(\mathrm{GNamax} / \mathrm{Cm}) *\left(\mathrm{y}(2)^{\wedge} 3\right)^{*} \mathrm{y}(3) *(\mathrm{y}(1)-$ $\mathrm{Na}$ _Battery)+Current(t,pulse_width,pulse_amp)/Cm; $(\mathrm{mf}-\mathrm{y}(2)) /(\operatorname{taum} / 1000)$; (hf-y $(3)) /(\operatorname{tauh} / 1000)$; $(\mathrm{nf}-\mathrm{y}(4)) /(\operatorname{taun} / 1000)]$; 